\title{
Existence of positive solutions of four-point BVPs for one-dimensional generalized Lane-Emden systems on whole line ${ }^{* \dagger}$
}

\author{
Pinghua Yang ${ }^{1}$ and Yuji Liu²
}

\begin{abstract}
${ }^{1}$ Department of Basic Courses, Shijiazhuang Mechanical Engineering College, Shijiazhuang 050000, P.R.China ${ }^{2}$ Department of Mathematics, Guangdong University of Finance and Economics, Guangzhou 510320, P.R.China E-mail: yujiliu@126.com, liuyuji888@sohu.com
\end{abstract}

\begin{abstract}
This paper is concerned with four-point boundary value problems of the one-dimensional generalized Lane-Emden systems on whole lines. The Green's functions $G(t, s)$ for the problem $-\left(\rho(t) x^{\prime}(t)\right)^{\prime}=0$ with boundary conditions $\lim _{t \rightarrow-\infty} x(t)-k x(\xi)=\lim _{t \rightarrow+\infty} x(t)-l x(\eta)=0$ and $\lim _{t \rightarrow-\infty} x(t)-k x(\xi)=\lim _{t \rightarrow+\infty} \rho(t) x^{\prime}(t)-l \rho(\eta) x^{\prime}(\eta)=0$ are obtained respectively. We proved that $G(t, s) \geq 0$ under some assumptions which actually generalize a corresponding result in [J. Math. Anal. Appl. 305 (2005) 253-276]. Sufficient conditions to guarantee the existence of positive solutions of this kind of models are established. Examples are given at the end of the paper.
\end{abstract}

2010 Mathematics Subject Classification. 34B10. 34B15, 35B10

Keywords. One-dimensional generalized Lane-Emden system, four-point boundary value problem, positive solution, fixed point theorem.

\section{Introduction}

It is well known that the following systems are called homogeneous and non-homogeneous LaneEmden systems, respectively,

$$
\left\{\begin{array}{l}
-\Delta u(x)=v^{p}(x), x \in \Omega \\
-\Delta v(x)=u^{q}(x), x \in \Omega \\
u(x)>0, v(x)>0, x \in \Omega \\
u(x)=v(x)=0, x \in \partial \Omega
\end{array}\right.
$$

and

$$
\left\{\begin{array}{l}
-\Delta u(x)=v^{p}(x)+\lambda f(x), x \in \Omega \\
-\Delta v(x)=u^{q}(x)+\lambda g(x), x \in \Omega \\
u(x)>0, v(x)>0, x \in \Omega \\
u(x)=v(x)=0, x \in \partial \Omega
\end{array}\right.
$$

where $p, q \in(1,+\infty), \Omega$ is a domain in the n-dimensional Euclidean space $R^{n}, \Delta=\sum_{i=1}^{n} \frac{\partial^{2}}{\partial x_{i}^{2}}$. LaneEmden systems (1.2) and (1.2) arise naturally from the study of various nonlinear phenomena, such as pattern formation, population evolution, chemical reaction, and so on see [28], and has attracted

\footnotetext{
* Supported by Natural Science Foundation of Guangdong province (No:7004569) and Natural Science Foundation of Hunan province, P.R.China(No:06JJ50008)

${ }^{\dagger}$ Corresponding author: Yuji Liu
} 
considerable attention in recent years. In the literature, properties of solutions to (1.1), such as: a priori estimate; existence results; Liouville type theorems; and some symmetric and uniqueness results, were obtained, see $[11,12]$. Multiplicity results have also been proved for problem (1.2) under certain conditions see [17].

The one-dimensional cases of problems (1.1) and (1.2) are the following forms, respectivly:

$$
\left\{\begin{array}{l}
-u^{\prime \prime}(t)=v^{p}(t)+\lambda f(t), t \in(-l, l), \\
-v^{\prime \prime}(t)=u^{q}(t)+\lambda g(t), t \in(-l, l) \\
u(t)>0, v(t)>0, t \in(-l, l) \\
u(-l)=v(-l)=u(l)=v(l)=0
\end{array}\right.
$$

and

$$
\left\{\begin{array}{l}
-u^{\prime \prime}(t)=v^{p}(t), t \in(-l, l) \\
-v^{\prime \prime}(t)=u^{q}(t), t \in(-l, l) \\
u(t)>0, v(t)>0, t \in(-l, l) \\
u(-l)=v(-l)=u(l)=v(l)=0
\end{array}\right.
$$

The multiplicity of positive solutions of problem (1.4) were studied in $[13,12]$ by making use of the nondegeneracy and uniqueness of solutions of problem (1.3).

The asymptotic theory of ordinary differential equations is an area in which there is great activity among a large number of investigators. In this theory, it is of great interest to investigate, in particular, the existence of solutions with prescribed asymptotic behavior, which are global in the sense that they are solutions on the whole line. The existence of global solutions with prescribed asymptotic behavior is usually formulated as the existence of solutions of boundary value problems on the whole line.

Motivated by mentioned papers, we consider the following four-point boundary value problem for second order singular differential system on the whole line

$$
\left\{\begin{array}{l}
{\left[\rho(t) x^{\prime}(t)\right]^{\prime}+f\left(t, x(t), y(t), x^{\prime}(t), y^{\prime}(t)\right)=0, a . e ., t \in \mathbb{R},} \\
{\left[\varrho(t) y^{\prime}(t)\right]^{\prime}+g\left(t, x(t), y(t), x^{\prime}(t), y^{\prime}(t)\right)=0, \text { a.e., } t \in \mathbb{R},} \\
\lim _{t \rightarrow-\infty} x(t)-a x(\xi)=0 \\
\lim _{t \rightarrow+\infty} x(t)-b x(\eta)=0 \\
\lim _{t \rightarrow-\infty} y(t)-c y(\xi)=0 \\
\lim _{t \rightarrow+\infty} \varrho(t) y^{\prime}(t)-d \varrho(\eta) y^{\prime}(\eta)=0
\end{array}\right.
$$

where

(a) $-\infty<\xi<\eta<+\infty, a, b, c, d \geq 0$ are constants,

(b) $f, g$ are nonnegative $(\rho, \varrho)$-Carathéodory functions see Definition 2.2, and $f(t, c, y, 0, v) \not \equiv 0$, $g(t, x, c, u, 0) \not \equiv 0$ on each subinterval of $\mathbb{R}$,

(c) $\mathbb{R}^{+}=[0, \infty), \rho, \varrho$ are nonnegative and continuous at almost every points on $\mathbb{R}$ and may be singular at several points on $\mathbb{R}$.

Nonlocal boundary value problems for ordinary differential equations (ODEs) was initiated by Il'in and Moiseev [18]. Since then, more general nonlocal boundary value problems (BVPs) were studied by several authors, see the text books $[1,14,16]$, the papers $[26]$, and the survey papers $[21,22]$ and the references cited there. However, the study on existence of positive solutions of 
nonlocal boundary value problems for differential equations on whole real lines does not seem to be sufficiently developed $[2,4,3,5,6,27]$ and the references therein.

In recent years, the existence of solutions of boundary value problems of the differential equations on the whole lines has been studied by many authors, see $[9,10,15,23,24,6,8,7,20]$ and the references therein. It is easy to see that $\operatorname{BVP}(1.5)$ is a generalized form of $\operatorname{BVP}(1.3)$ and (1.4). We call (1.5) a one-dimensional generalized Lane-Emden system since it is actually a generalization of (1.3) and (1.4).

A function $x: \mathbb{R} \rightarrow \mathbb{R}$ is called a solution of $\operatorname{BVP}(1.5)$ if $x \in C(\mathbb{R}), \rho x^{\prime}$ is continuous at almost all points on $\mathbb{R}$ (except several removable discontinuity points), $\left(\rho(t) x^{\prime}(t)\right)^{\prime}$ exists at almost all points on $\mathbb{R}$ and is measurable on $\mathbb{R}$ and all equations in (1.5) are satisfied.

The purpose is to establish sufficient conditions for the existence of positive solutions of BVP(1.5). Our results and methods are different from those in $[3,4,7,27,6,2,8]$.

The remainder of this paper is organized as follows: the preliminary results are given in Section 2 , the existence result of positive solutions of $\operatorname{BVP}(1.5)$ is proved in Section 3. In Section 4, an example is given to illustrate the efficiency of the main result.

\section{Preliminary results}

In this section, we present some background definitions in Banach spaces see [14]. A Banach space is proved and the relatively compact properties of subsets of the Banach space are described. The preliminary results are given too.

Definition 2.1. Let $X$ and $Y$ be Banach spaces. An operator $T: X \rightarrow Y$ is completely continuous if it is continuous and maps bounded sets into relatively compact sets.

Definition 2.2. $F$ is called a $(\rho, \varrho)$-Carathédory function, that is

(i) $t \rightarrow F\left(t, x_{1}, x_{2}, \frac{x_{3}}{\rho(t)}, \frac{x_{4}}{\varrho(t)}\right)$ is defined almost every point on $\mathbb{R}$ and is measurable on $R$ for each $x_{1}, x_{2}, x_{3}, x_{4} \in R$,

(ii) $\left(x_{1}, x_{2}, x_{3}, x_{4}\right) \rightarrow F\left(t, x_{1}, x_{2}, \frac{x_{3}}{\rho(t)}, \frac{x_{4}}{\varrho(t)}\right)$ is continuous on $\mathbb{R}^{4}$ for almost every $t \in R$,

(iii) for each $r>0$, there exists nonnegative function $\varphi_{r} \in L^{1}(\mathbb{R})$ such that $\left|x_{i}\right| \leq r(i=1,2,3,4)$ implies

$$
\left|F\left(t, x_{1}, x_{2}, \frac{x_{3}}{\rho(t)}, \frac{x_{4}}{\varrho(t)}\right)\right| \leq \varphi_{r}(t) \text {, a.e.t } \in \mathbb{R} \text {. }
$$

Suppose that

$$
\begin{gathered}
\int_{-\infty}^{+\infty} \frac{d u}{\rho(u)}<+\infty, \int_{-\infty}^{+\infty} \frac{d u}{\varrho(u)}<+\infty \\
(1-a) \int_{-\infty}^{+\infty} \frac{d u}{\rho(u)}+a \int_{-\infty}^{\xi} \frac{d u}{\rho(u)} \geq 0 \\
\int_{\eta}^{+\infty} \frac{d u}{\rho(u)}+(1-b) \int_{-\infty}^{\eta} \frac{d u}{\rho(u)} \geq 0, \\
\Delta=:(1-a) \int_{-\infty}^{+\infty} \frac{d u}{\rho(u)}-(1-a) b \int_{-\infty}^{\eta} \frac{d u}{\rho(u)}+a(1-b) \int_{-\infty}^{\xi} \frac{d u}{\rho(u)}>0, \\
c<1, d<1 .
\end{gathered}
$$


For a function $\varphi: \mathbb{R} \rightarrow \mathbb{R}$, denote $\|\varphi\|_{0}=\sup _{t \in \mathbb{R}}|\varphi(t)|$ and $\|\varphi\|_{1}=\int_{-\infty}^{+\infty}|\varphi(s)| d s$ if they exist. Choose

$$
X=\left\{x: \mathbb{R} \rightarrow \mathbb{R}: \begin{array}{c}
x \in C(\mathbb{R}), \rho x^{\prime} \in C(\mathbb{R}), \lim _{t \rightarrow-\infty} x(t), \lim _{t \rightarrow+\infty} x(t) \\
\lim _{t \rightarrow-\infty} \rho(t) x^{\prime}(t), \lim _{t \rightarrow+\infty} \rho(t) x^{\prime}(t) \text { exsit and are finite }
\end{array}\right\}
$$

For $x \in X$, define

$$
\|x\|_{X}=\max \left\{\|x\|_{0},\left\|\rho \mid x^{\prime}\right\|_{0}\right\} .
$$

Lemma 2.1. $X$ is a Banach space with $\|\cdot\|_{X}$ defined.

Proof. It is easy to see that $X$ is a normed linear space. Let $\left\{x_{u}\right\}$ be a Cauchy sequence in $X$. Then $\left\|x_{u}-x_{v}\right\| \rightarrow 0, u, v \rightarrow+\infty$. It follows that

$$
\begin{aligned}
& \lim _{t \rightarrow-\infty} x_{u}(t), \lim _{t \rightarrow+\infty} x_{u}(t), \lim _{t \rightarrow-\infty} \rho(t) x_{u}^{\prime}(t), \lim _{t \rightarrow+\infty} \rho(t) x_{u}^{\prime}(t) \text { exist }, \\
& \left\|x_{u}-x_{v}\right\|_{0} \rightarrow 0, u, v \rightarrow+\infty \\
& \| \rho\left(x_{u}^{\prime}-x_{v}^{\prime} \|_{0}, u, v \rightarrow+\infty .\right.
\end{aligned}
$$

Thus there exists two functions $x_{0}, y_{0}$ defined on $R$ such that

$$
\lim _{u \rightarrow+\infty} x_{u}(t)=x_{0}(t), \lim _{u \rightarrow+\infty} \rho(t) x_{u}^{\prime}(t)=y_{0}(t) .
$$

It follows that

$$
\begin{aligned}
& \left\|x_{u}-x_{0}\right\|_{0} \rightarrow 0, u \rightarrow+\infty, \\
& \left\|\rho x_{u}^{\prime}(t)-y_{0}\right\|_{0} \rightarrow 0, u \rightarrow+\infty .
\end{aligned}
$$

This means that functions $x_{0}, y_{0}: \mathbb{R} \rightarrow \mathbb{R}$ are well defined.

Step 1. Prove that $x_{0}, y_{0} \in C(\mathbb{R})$.

We have for $t_{0} \in R$ that

$$
\begin{aligned}
& \left|x_{0}(t)-x_{0}\left(t_{0}\right)\right| \leq\left|x_{0}(t)-x_{N}(t)\right|+\left|x_{N}(t)-x_{N}\left(t_{0}\right)\right|+\left|x_{N}\left(t_{0}\right)-x_{0}\left(t_{0}\right)\right| \\
& \leq 2|| x_{N}-x_{0} \|_{0}+\left|x_{N}(t)-x_{N}\left(t_{0}\right)\right| .
\end{aligned}
$$

Since $\left\|x_{u}-x_{0}\right\|_{0} \rightarrow 0, u \rightarrow+\infty$ and $x_{u}(t)$ is continuous on $\mathbb{R}$, then for any $\varepsilon>0$ we can choose $N$ and $\delta>0$ such that ||$x_{N}-x_{0} \|_{0}<\varepsilon$ and $\left|x_{N}(t)-x_{N}\left(t_{0}\right)\right|<\varepsilon$ for all $\left|t-t_{0}\right|<\delta$. Thus $\left|x_{0}(t)-x_{0}\left(t_{0}\right)\right|<3 \varepsilon$ for all $\left|t-t_{0}\right|<\delta$. So $x_{0} \in C(\mathbb{R})$. Similarly we can prove that $y_{0} \in C(\mathbb{R})$.

Step 2. Prove that the limits $\lim _{t \rightarrow-\infty} x_{0}(t), \lim _{t \rightarrow+\infty} x_{0}(t), \lim _{t \rightarrow-\infty} y_{0}(t), \lim _{t \rightarrow+\infty} y_{0}(t)$ exist.

Suppose that $\lim _{t \rightarrow-\infty} x_{u}(t)=A_{u}^{-}$. By $\left\|x_{u}-x_{v}\right\|_{0} \rightarrow 0, u, v \rightarrow+\infty$, we know that $A_{u}^{-}$is a Cauchy sequence. Then $\lim _{u \rightarrow+\infty} A_{u}^{-}$exists. By $\left\|x_{u}-x_{0}\right\|_{0} \rightarrow 0, u \rightarrow+\infty$, we get that

$$
\lim _{t \rightarrow-\infty} x_{0}(t)=\lim _{t \rightarrow-\infty} \lim _{u \rightarrow+\infty} x_{u}(t)=\lim _{u \rightarrow+\infty} \lim _{t \rightarrow-\infty} x_{u}(t)=\lim _{u \rightarrow+\infty} A_{u}^{-} .
$$

Hence $\lim _{t \rightarrow-\infty} x_{0}(t)$ exists. Similarly we can prove that $\lim _{t \rightarrow+\infty} x_{0}(t), \lim _{t \rightarrow-\infty} y_{0}(t), \lim _{t \rightarrow+\infty} y_{0}(t)$ exist. 
Step 3. Prove that $y_{0}(t)=\rho(t) x_{0}^{\prime}(t)$.

We have for some $c_{u} \in R$ that

$$
\begin{aligned}
& \left|x_{u}(t)-\lim _{t \rightarrow-\infty} x_{u}(t)-\int_{-\infty}^{t} \frac{y_{0}(s)}{\rho(s)} d s\right|=\left|\int_{-\infty}^{t} x_{u}^{\prime}(s) d s-\int_{-\infty}^{t} \frac{y_{0}(s)}{\rho(s)} d s\right| \\
& \leq \int_{-\infty}^{t}\left|x_{u}^{\prime}(s)-\frac{y_{0}(s)}{\rho(s)}\right| d s \leq \int_{-\infty}^{t} \frac{1}{\rho(t)} d s \sup _{t \in R}\left|\rho(t) x_{u}^{\prime}(t)-y_{0}(t)\right| \\
& \leq \int_{-\infty}^{+\infty} \frac{1}{\rho(t)} d s\left\|\rho x_{u}^{\prime}-y_{0}\right\|_{0} \rightarrow 0 \text { as } u \rightarrow+\infty
\end{aligned}
$$

So $\lim _{u \rightarrow+\infty}\left(x_{u}(t)-\lim _{t \rightarrow-\infty} x_{u}(t)\right)=\int_{-\infty}^{t} \frac{y_{0}(s)}{\rho(s)} d s$. Then $x_{0}(t)-c_{0}=\int_{-\infty}^{t} \frac{y_{0}(s)}{\rho(s)} d s$. It follows that $\frac{y_{0}(t)}{\rho(t)}=x_{0}^{\prime}(t)$. So $x_{0} \in X$ with $x_{u} \rightarrow x_{0}$ as $u \rightarrow+\infty$. It follows that $X$ is a Banach space.

Lemma 2.2. Let $M$ be a subset of $X$. Then $M$ is relatively compact if and only if the following conditions are satisfied:

(i) both $\{x: x \in M\}$ and $\left\{t \rightarrow \rho(t) x^{\prime}(t): x \in M\right\}$ are uniformly bounded, in $\mathbb{R}$,

(ii) both $\{x: x \in M\}$ and $\left\{t \rightarrow \rho(t) x^{\prime}(t): x \in M\right\}$ are equicontinuous in any subinterval $[a, b]$

(iii) both $\{x: x \in M\}$ and $\left\{t \rightarrow \rho(t) x^{\prime}(t): x \in M\right\}$ are equi-convergent as $t \rightarrow \pm \infty$.

Proof. " $\Leftarrow "$. From Lemma 2.1, we know $X$ is a Banach space. In order to prove that the subset $M$ is relatively compact in $X$, we only need to show $M$ is totally bounded in $X$, that is for all $\varepsilon>0, M$ has a finite $\varepsilon$-net.

For any given $\varepsilon>0$, by (i) and (iii), there exist constants $A_{x}, C_{x}, T>0, a>0$, we have

$$
\begin{aligned}
& \left|x(t)-A_{x}\right| \leq \frac{\varepsilon}{3},\left|\rho(t) x^{\prime}(t)-C_{x}\right|<\frac{\varepsilon}{3}, t \leq-T, x \in M, \\
& \left|x\left(t_{1}\right)-x\left(t_{2}\right)\right| \leq \frac{\varepsilon}{3},\left|\rho\left(t_{1}\right) x^{\prime}\left(t_{1}\right)-\rho\left(t_{2}\right) x^{\prime}\left(t_{2}\right)\right|<\frac{\varepsilon}{3}, t_{1}, t_{2} \geq T, x \in M, \\
& \left|x\left(t_{1}\right)-x\left(t_{2}\right)\right| \leq \frac{\varepsilon}{3},\left|\rho\left(t_{1}\right) x^{\prime}\left(t_{1}\right)-\rho\left(t_{2}\right) x^{\prime}\left(t_{2}\right)\right|<\frac{\varepsilon}{3}, t_{1}, t_{2} \leq-T, x \in M .
\end{aligned}
$$

For $T>0$, define $\left.X\right|_{[-T, T]}=\left\{x: x, \rho(t) x^{\prime} \in C[-T, T]\right\}$. For $\left.x \in X\right|_{[-T, T]}$, define

$$
\|x\|_{T}=\max \left\{\max _{t \in[-T, T]}|x(t)|, \max _{t \in[-T, T]} \rho(t)\left|x^{\prime}(t)\right|\right\} .
$$

Similarly to Claim 2.1, we can prove that $X_{[-T, T]}$ is a Banach space.

Let $\left.M\right|_{[-T, T]}=\{t \rightarrow x(t), t \in[-T, T]: x \in M\}$. Then $\left.M\right|_{[-T, T]}$ is a subset of $\left.X\right|_{[-T, T]}$. By (i) and (ii), and Ascoli-Arzela theorem, we can know that $\left.M\right|_{[-T, T]}$ is relatively compact. Thus, there exist $x_{1}, x_{2}, \cdots, x_{k} \in M$ such that, for any $x \in M$, we have that there exists some $i=1,2, \cdots, k$ such that

$$
\left\|x-x_{i}\right\|_{T}=\max \left\{\sup _{t \in[-T, T]}\left|x(t)-x_{i}(t)\right|, \sup _{t \in[-T, T]} \rho(t)\left|x^{\prime}(t)-x_{i}^{\prime}(t)\right|\right\} \leq \frac{\varepsilon}{3} .
$$


Therefore, for $x \in M$, we have that

$$
\begin{aligned}
& || x-x_{i}||_{X}=\max \left\{\sup _{t \in[-T, T]}\left|x(t)-x_{i}(t)\right|, \sup _{t \in[-T, T]} \rho(t)\left|x^{\prime}(t)-x_{i}^{\prime}(t)\right|,\right. \\
& \left.\sup _{t \geq T}\left|x(t)-x_{i}(t)\right|, \sup _{t \geq T} \rho(t)\left|x^{\prime}(t)-x_{i}^{\prime}(t)\right| \sup _{t \leq-T}\left|x(t)-x_{i}(t)\right|, \sup _{t \leq-T} \rho(t)\left|x^{\prime}(t)-x_{i}^{\prime}(t)\right|\right\} \\
& \leq \max \left\{\frac{\varepsilon}{3}, \sup _{t \geq T}|x(t)-x(T)|+\sup _{t \geq T}\left|x(T)-x_{i}(T)\right|+\sup _{t \geq T}\left|x_{i}(T)-x_{i}(t)\right|,\right. \\
& \sup _{t \geq T}\left|\rho(t) x^{\prime}(t)-\rho(T) x^{\prime}(T)\right|+\sup _{t \geq T}\left|\rho(T) x^{\prime}(T)-\rho(T) x_{i}^{\prime}(T)\right|+\sup _{t \geq T}\left|\rho(T) x_{i}^{\prime}(T)-\rho(t) x_{i}^{\prime}(t)\right| \\
& \sup _{t \leq-T}|x(t)-x(T)|+\sup _{t \leq-T}\left|x(T)-x_{i}(T)\right|+\sup _{t \leq-T}\left|x_{i}(T)-x_{i}(t)\right|, \\
& \left.\sup _{t \leq-T}\left|\rho(t) x^{\prime}(t)-\rho(T) x^{\prime}(T)\right|+\sup _{t \leq-T}\left|\rho(T) x^{\prime}(T)-\rho(T) x_{i}^{\prime}(T)\right|+\sup _{t \leq-T}\left|\rho(T) x_{i}^{\prime}(T)-\rho(t) x_{i}^{\prime}(t)\right|\right\} \leq \varepsilon .
\end{aligned}
$$

So, for any $\varepsilon>0, M$ has a finite $\varepsilon$-net $\left\{U_{x_{1}}, U_{x_{2}}, \cdots, U_{x_{k}}\right\}$, that is, $M$ is totally bounded in $X$. Hence $M$ is relatively compact in $X$.

$\Rightarrow$. Assume that $M$ is relatively compact, then for any $\varepsilon>0$, there exists a finite $\varepsilon$-net of $M$. Let the finite $\varepsilon$-net be $\left\{U_{x_{1}}, U_{x_{2}}, \cdots, U_{x_{k}}\right\}$ with $x_{i} \subset M$. Then for any $x \in M$, there exists $U_{x_{i}}$ such that $x \in U_{x_{i}}$ and

$$
\begin{aligned}
& |x(t)| \leq\left|x(t)-x_{i}(t)\right|+\left|x_{i}(t)\right| \leq \varepsilon+\max \left\{\sup _{t \in R}\left|x_{i}(t)\right|: i=1,2, \cdots, k\right\}, \\
& \rho(t)\left|x^{\prime}(t)\right| \leq \varepsilon+\max \left\{\sup _{t \in \mathbb{R}} \rho(t)\left|x_{i}^{\prime}(t)\right|: i=1,2, \cdots, k\right\} .
\end{aligned}
$$

It follows that both $M$ and $\left\{\rho(t) x^{\prime}: x \in M\right\}$ are uniformly bounded. Then (i) holds. Furthermore, there exists $T>0$ such that $\left|x_{i}\left(t_{1}\right)-x_{i}\left(t_{2}\right)\right|<\varepsilon$ for all $t_{1}, t_{2} \geq T$ and all $t_{1}, t_{2} \leq-T$ and $i=1,2, \cdots, k$. Then we have for $t_{1}, t_{2} \in R$ that

$$
\begin{aligned}
& \left|x\left(t_{1}\right)-x\left(t_{2}\right)\right| \leq\left|x\left(t_{1}\right)-x_{i}\left(t_{1}\right)\right|+\left|x_{i}\left(t_{1}\right)-x_{i}\left(t_{2}\right)\right|+\left|x_{i}\left(t_{2}\right)-x\left(t_{2}\right)\right| \\
& \leq 3 \varepsilon \text { for all } t_{1}, t_{2} \geq T, t_{1}, t_{2} \leq-T, x \in M .
\end{aligned}
$$

Similarly we have that $\left|\rho\left(t_{1}\right) x^{\prime}\left(t_{1}\right)-\rho\left(t_{2}\right) x^{\prime}\left(t_{2}\right)\right| \leq 3 \varepsilon$ for all $t_{1}, t_{2} \geq T, t_{1}, t_{2} \leq-T, x \in M$. Thus (iii) is valid. Similarly we can prove that (ii) holds. Consequently, the proof is proved.

Choose

$$
Y=\left\{y: \mathbb{R} \rightarrow \mathbb{R}: \begin{array}{c}
y \in C(\mathbb{R}), \varrho y^{\prime} \in C(\mathbb{R}), \lim _{t \rightarrow-\infty} y(t), \lim _{t \rightarrow+\infty} y(t) \\
\lim _{t \rightarrow-\infty} \varrho(t) x^{\prime}(t), \lim _{t \rightarrow+\infty} \varpi(t) x^{\prime}(t) \text { exist and are finite }
\end{array}\right\} .
$$


For $y \in Y$, define $\|y\|_{Y}=\max \left\{\|y\|_{0},\left\|\varrho y^{\prime}\right\|_{0}\right\}$. Let $E=X \times Y$ be defined with the norm $\|(x, y)\|=\max \left\{\|x\|_{X},\|y\|_{Y}\right\}$.

Lemma 2.3. $Y$ is a Banach space with $\|\cdot\|_{Y}$ defined. $E$ is a Banach space.

Proof. Similarly to Lemma 2.1 and the proof is omitted.

Lemma 2.4. Let $M$ be a subset of $Y$. Then $M$ is relatively compact if and only if the following conditions are satisfied:

(ii) both $\{y: y \in M\}$ and $\left\{t \rightarrow \varrho(t) y^{\prime}(t): y \in M\right\}$ are uniformly bounded,

(ii) both $\{y: y \in M\}$ and $\left\{t \rightarrow \varrho(t) y^{\prime}(t): y \in M\right\}$ are equicontinuous in any subinterval $[a, b]$ in $\mathbb{R}$,

(ii) both $\{y: y \in M\}$ and $\left\{t \rightarrow \varrho(t) y^{\prime}(t): y \in M\right\}$ are equi-convergent as $t \rightarrow \pm \infty$.

Proof. Similarly to Lemma 2.2, we omit the details.

Denote

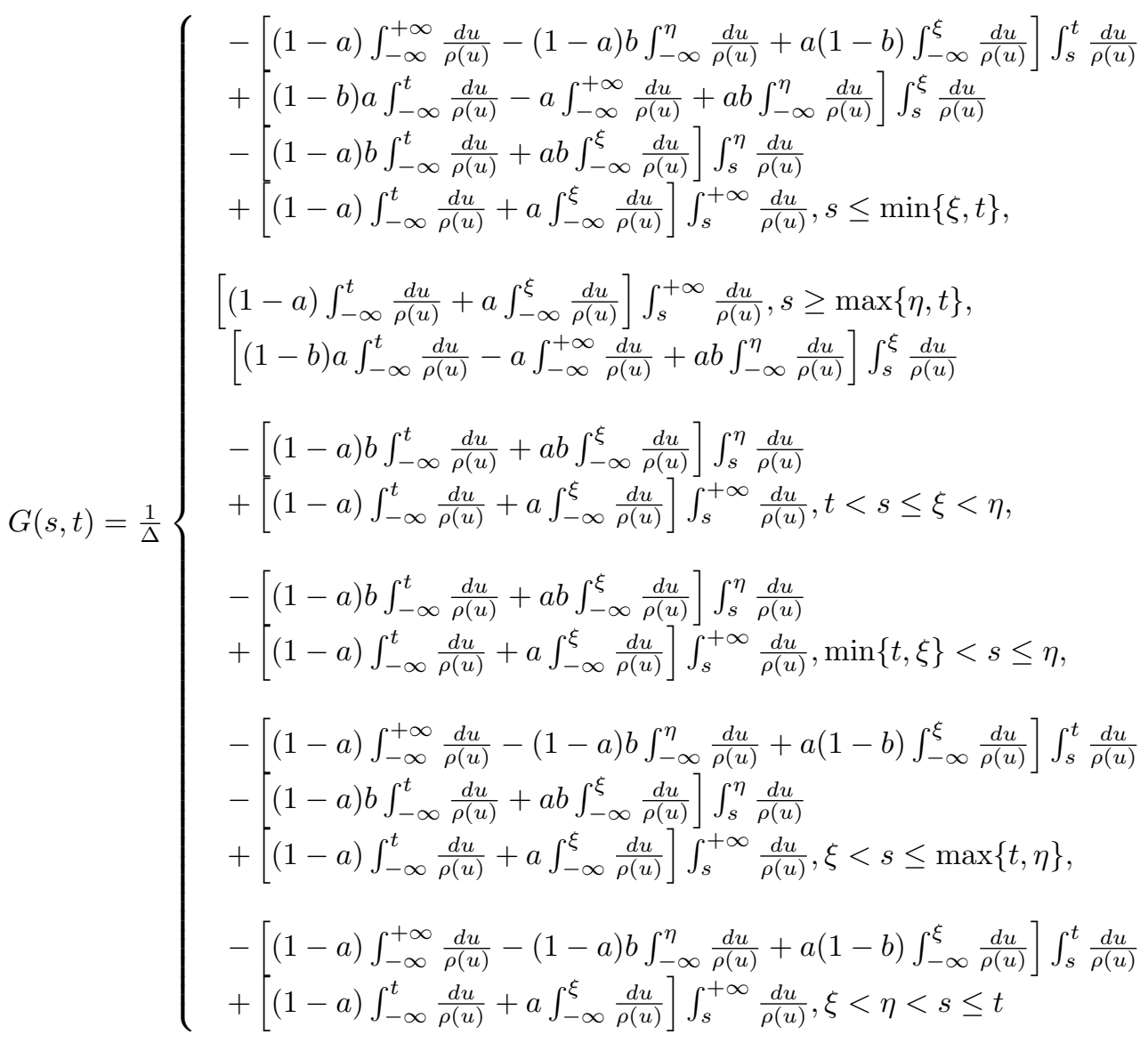


and

$$
H(s, t)=\frac{1}{(1-c)(1-d)}\left\{\begin{array}{l}
c(1-d) \int_{-\infty}^{s} \frac{d u}{\varrho(u)}+(1-c)(1-d) \int_{-\infty}^{t} \frac{d u}{\varrho(u)}, s \leq \min \{\xi, t\}, \\
c \int_{-\infty}^{\xi} \frac{d u}{\varrho(u)}+(1-c) \int_{-\infty}^{t} \frac{d u}{\varrho(u)}, s \geq \max \{\eta, t\}, \\
(1-d)(1-c) \int_{-\infty}^{t} \frac{d u}{\varrho(u)}+c(1-d) \int_{-\infty}^{s} \frac{d u}{\varrho(u)}, t<s \leq \xi<\eta, \\
c(1-d) \int_{-\infty}^{\xi} \frac{d u}{\varrho(u)}+(1-c)(1-d) \int_{-\infty}^{t} \frac{d u}{\varrho(u)}, \min \{t, \xi\}<s \leq \eta, \\
c(1-d) \int_{-\infty}^{\xi} \frac{d u}{\varrho(u)}, \xi<s \leq \max \{t, \eta\}, \\
c \int_{-\infty}^{\xi} \frac{d u}{\varrho(u)}+(1-c)(1-d) \int_{-\infty}^{t} \frac{d u}{\varrho(u)}, \xi<\eta<s \leq t .
\end{array}\right.
$$

Lemma 2.4. Suppose that (2.7), (2.8.1), (2.8.2), (2.8.3) and (2.8) hold. Then $(x, y) \in E$ is a solution of $\operatorname{BVP}(1.5)$ if and only if $(x, y) \in E$ and

$$
\left\{\begin{array}{l}
x(t)=\int_{-\infty}^{+\infty} G(t, s) f\left(s, x(s), y(s), x^{\prime}(s), y^{\prime}(s)\right) d s \\
y(t)=\int_{-\infty}^{+\infty} H(t, s) g\left(s, x(s), y(s), x^{\prime}(s), y^{\prime}(s)\right) d s
\end{array}\right.
$$

Proof. Since $(x, y) \in E, f, g$ are Caratheodory functions, then

$$
\|(x, y)\|=\max \left\{\sup _{t \in R}|x(t)|, \sup _{t \in R} \rho(t)\left|x^{\prime}(t)\right|, \sup _{t \in R}|y(t)|, \sup _{t \in R} \varrho(t)\left|y^{\prime}(t)\right|\right\}=r<+\infty,
$$

and $\int_{-\infty}^{+\infty} f\left(r, x(r), y(r), x^{\prime}(r), y^{\prime}(r)\right) d r$ and $\int_{-\infty}^{+\infty} g\left(r, x(r), y(r), x^{\prime}(r), y^{\prime}(r)\right) d r$ converge. If $(x, y)$ is a solution of $\operatorname{BVP}(1.5)$, we get from $\left[\rho(t) x^{\prime}(t)\right]^{\prime}+f\left(t, x(t), y(t), x^{\prime}(t), y^{\prime}(t)\right)=0$ that there exist constants $A, B \in \mathbb{R}$ such that

$$
\begin{aligned}
& \rho(t) x^{\prime}(t)=A-\int_{-\infty}^{t} f\left(s, x(s), y(s), x^{\prime}(s), y^{\prime}(s)\right) d s . \\
& x(t)=B+A \int_{-\infty}^{t} \frac{d u}{\rho(u)}-\int_{-\infty}^{t} \frac{1}{\rho(s)} \int_{-\infty}^{s} f\left(u, x(u), y(u), x^{\prime}(u), y^{\prime}(u)\right) d u d s .
\end{aligned}
$$

Since

$$
\begin{aligned}
& \int_{-\infty}^{t} \frac{1}{\rho(s)} \int_{-\infty}^{s} f\left(u, x(u), y(u), x^{\prime}(u), y^{\prime}(u)\right) d u d s \\
& =\int_{-\infty}^{t} \int_{-\infty}^{s} f\left(u, x(u), y(u), x^{\prime}(u), y^{\prime}(u)\right) d u d\left(\int_{-\infty}^{s} \frac{d u}{\rho(u)}\right) \\
& =\left.\int_{-\infty}^{s} f\left(u, x(u), y(u), x^{\prime}(u), y^{\prime}(u)\right) d u\left(\int_{-\infty}^{s} \frac{d u}{\rho(u)}\right)\right|_{-\infty} ^{t} \\
& -\int_{-\infty}^{t} \int_{-\infty}^{s} \frac{d u}{\rho(u)} f\left(s, x(s), y(s), x^{\prime}(s), y^{\prime}(s)\right) d s \\
& =\int_{-\infty}^{t}\left(\int_{s}^{t} \frac{d u}{\rho(u)}\right) f\left(s, x(s), y(s), x^{\prime}(s), y^{\prime}(s)\right) d s .
\end{aligned}
$$


Then

$$
x(t)=B+A \int_{-\infty}^{t} \frac{d u}{\rho(u)}-\int_{-\infty}^{t}\left(\int_{s}^{t} \frac{d u}{\rho(u)}\right) f\left(s, x(s), y(s), x^{\prime}(s), y^{\prime}(s)\right) d s .
$$

From $\lim _{t \rightarrow-\infty} x(t)-a x(\xi)=0$ and $\lim _{t \rightarrow+\infty} x(t)-b x(\eta)=0$, we have

$$
\begin{gathered}
(1-a) B-a \int_{-\infty}^{\xi} \frac{d u}{\rho(u)} A=-a \int_{-\infty}^{\xi}\left(\int_{s}^{\xi} \frac{d u}{\rho(u)}\right) f\left(s, x(s), y(s), x^{\prime}(s), y^{\prime}(s)\right) d s \\
(1-b) B+\left(\int_{-\infty}^{+\infty} \frac{d u}{\rho(u)}-b \int_{-\infty}^{\eta} \frac{d u}{\rho(u)}\right) A=\int_{-\infty}^{+\infty}\left(\int_{s}^{+\infty} \frac{d u}{\rho(u)}\right) f\left(s, x(s), y(s), x^{\prime}(s), y^{\prime}(s)\right) d s \\
\quad-b \int_{-\infty}^{\eta}\left(\int_{s}^{\eta} \frac{d u}{\rho(u)}\right) f\left(s, x(s), y(s), x^{\prime}(s), y^{\prime}(s)\right) d s .
\end{gathered}
$$

It follows that

$$
\begin{aligned}
& A=\frac{1}{\Delta}\left[-(1-a) b \int_{-\infty}^{\eta}\left(\int_{s}^{\eta} \frac{d u}{\rho(u)}\right) f\left(s, x(s), y(s), x^{\prime}(s), y^{\prime}(s)\right) d s\right. \\
& +(1-a) \int_{-\infty}^{+\infty}\left(\int_{s}^{+\infty} \frac{d u}{\rho(u)}\right) f\left(s, x(s), y(s), x^{\prime}(s), y^{\prime}(s)\right) d s \\
& \left.+(1-b) a \int_{-\infty}^{\xi}\left(\int_{s}^{\xi} \frac{d u}{\rho(u)}\right) f\left(s, x(s), y(s), x^{\prime}(s), y^{\prime}(s)\right) d s\right], \\
& B=\frac{1}{\Delta}\left[-a\left(\int_{-\infty}^{+\infty} \frac{d u}{\rho(u)}-b \int_{-\infty}^{\eta} \frac{d u}{\rho(u)}\right) \int_{-\infty}^{\xi}\left(\int_{s}^{\xi} \frac{d u}{\rho(u)}\right) f\left(s, x(s), y(s), x^{\prime}(s), y^{\prime}(s)\right) d s\right. \\
& +a \int_{-\infty}^{\xi} \frac{d u}{\rho(u)}\left(-l \int_{-\infty}^{\eta}\left(\int_{s}^{\eta} \frac{d u}{\rho(u)}\right) f\left(s, x(s), y(s), x^{\prime}(s), y^{\prime}(s)\right) d s\right. \\
& \left.\left.+\int_{-\infty}^{+\infty}\left(\int_{s}^{+\infty} \frac{d u}{\rho(u)}\right) f\left(s, x(s), y(s), x^{\prime}(s), y^{\prime}(s)\right) d s\right)\right] .
\end{aligned}
$$

Substituting $A, B$ into (2.10), we get that

$$
\begin{aligned}
& x(t)=-\int_{-\infty}^{t}\left(\int_{s}^{t} \frac{d u}{\rho(u)}\right) f\left(s, x(s), y(s), x^{\prime}(s), y^{\prime}(s)\right) d s \\
& +\left[\frac{(1-b) a}{\Delta} \int_{-\infty}^{t} \frac{d u}{\rho(u)}-\frac{k}{\Delta} \int_{-\infty}^{+\infty} \frac{d u}{\rho(u)}+\frac{a b}{\Delta} \int_{-\infty}^{\eta} \frac{d u}{\rho(u)}\right] \int_{-\infty}^{\xi}\left(\int_{s}^{\xi} \frac{d u}{\rho(u)}\right) f\left(s, x(s), y(s), x^{\prime}(s), y^{\prime}(s)\right) d s \\
& -\left[\frac{(1-a) b}{\Delta} \int_{-\infty}^{t} \frac{d u}{\rho(u)}+\frac{a b}{\Delta} \int_{-\infty}^{\xi} \frac{d u}{\rho(u)}\right] \int_{-\infty}^{\eta}\left(\int_{s}^{\eta} \frac{d u}{\rho(u)}\right) f\left(s, x(s), y(s), x^{\prime}(s), y^{\prime}(s)\right) d s \\
& +\left[\frac{1-a}{\Delta} \int_{-\infty}^{t} \frac{d u}{\rho(u)}+\frac{a}{\Delta} \int_{-\infty}^{\xi} \frac{d u}{\rho(u)}\right] \int_{-\infty}^{+\infty}\left(\int_{s}^{+\infty} \frac{d u}{\rho(u)}\right) f\left(s, x(s), y(s), x^{\prime}(s), y^{\prime}(s)\right) d s .
\end{aligned}
$$

On sees from

$$
\Delta=:(1-a) \int_{-\infty}^{+\infty} \frac{d u}{\rho(u)}-(1-a) b \int_{-\infty}^{\eta} \frac{d u}{\rho(u)}+a(1-b) \int_{-\infty}^{\xi} \frac{d u}{\rho(u)}
$$


that the following cases are included:

(1) $t \leq \xi<\eta$. We have

$$
\begin{aligned}
& x(t)=\int_{-\infty}^{t}\left[-\left[(1-a) \int_{-\infty}^{+\infty} \frac{d u}{\rho(u)}-(1-a) l \int_{-\infty}^{\eta} \frac{d u}{\rho(u)}+a(1-b) \int_{-\infty}^{\xi} \frac{d u}{\rho(u)}\right] \int_{s}^{t} \frac{d u}{\rho(u)}\right. \\
& +\left[(1-b) a \int_{-\infty}^{t} \frac{d u}{\rho(u)}-a \int_{-\infty}^{+\infty} \frac{d u}{\rho(u)}+a b \int_{-\infty}^{\eta} \frac{d u}{\rho(u)}\right] \int_{s}^{\xi} \frac{d u}{\rho(u)} \\
& -\left[(1-a) b \int_{-\infty}^{t} \frac{d u}{\rho(u)}+a b \int_{-\infty}^{\xi} \frac{d u}{\rho(u)}\right] \int_{s}^{\eta} \frac{d u}{\rho(u)} \\
& \left.+\left((1-a) \int_{-\infty}^{t} \frac{d u}{\rho(u)}+a \int_{-\infty}^{\xi} \frac{d u}{\rho(u)}\right) \int_{s}^{+\infty} \frac{d u}{\rho(u)}\right] f\left(s, x(s), y(s), x^{\prime}(s), y^{\prime}(s)\right) d s \\
& +\int_{t}^{\xi}\left[-\left((1-a) b \int_{-\infty}^{t} \frac{d u}{\rho(u)}+a b \int_{-\infty}^{\xi} \frac{d u}{\rho(u)}\right) \int_{s}^{\eta} \frac{d u}{\rho(u)}\right. \\
& \left.+\left((1-a) \int_{-\infty}^{t} \frac{d u}{\rho(u)}+a \int_{-\infty}^{\xi} \frac{d u}{\rho(u)}\right) \int_{s}^{+\infty} \frac{d u}{\rho(u)}\right] f\left(s, x(s), y(s), x^{\prime}(s), y^{\prime}(s)\right) d s \\
& +\int_{\xi}^{\eta}\left[-\left((1-a) b \int_{-\infty}^{t} \frac{d u}{\rho(u)}+a b \int_{-\infty}^{\xi} \frac{d u}{\rho(u)}\right) \int_{s}^{\eta} \frac{d u}{\rho(u)}\right. \\
& \left.+\left((1-a) \int_{-\infty}^{t} \frac{d u}{\rho(u)}+a \int_{-\infty}^{\xi} \frac{d u}{\rho(u)}\right) \int_{s}^{+\infty} \frac{d u}{\rho(u)}\right] f\left(s, x(s), y(s), x^{\prime}(s), y^{\prime}(s)\right) d s \\
& +\int_{\eta}^{+\infty}\left[\left((1-a) \int_{-\infty}^{t} \frac{d u}{\rho(u)}+a \int_{-\infty}^{\xi} \frac{d u}{\rho(u)}\right) \int_{s}^{+\infty} \frac{d u}{\rho(u)}\right] f\left(s, x(s), y(s), x^{\prime}(s), y^{\prime}(s)\right) d s
\end{aligned}
$$

(2) $\xi \leq t \leq \eta$. We have

$$
\begin{aligned}
& x(t)=\int_{-\infty}^{\xi}\left[-\left[(1-a) \int_{-\infty}^{+\infty} \frac{d u}{\rho(u)}-(1-a) l \int_{-\infty}^{\eta} \frac{d u}{\rho(u)}+a(1-b) \int_{-\infty}^{\xi} \frac{d u}{\rho(u)}\right] \int_{s}^{t} \frac{d u}{\rho(u)}\right. \\
& +\left[(1-b) a \int_{-\infty}^{t} \frac{d u}{\rho(u)}-a \int_{-\infty}^{+\infty} \frac{d u}{\rho(u)}+a b \int_{-\infty}^{\eta} \frac{d u}{\rho(u)}\right] \int_{s}^{\xi} \frac{d u}{\rho(u)} \\
& -\left[(1-a) b \int_{-\infty}^{t} \frac{d u}{\rho(u)}+a b \int_{-\infty}^{\xi} \frac{d u}{\rho(u)}\right] \int_{s}^{\eta} \frac{d u}{\rho(u)} \\
& \left.+\left((1-a) \int_{-\infty}^{t} \frac{d u}{\rho(u)}+a \int_{-\infty}^{\xi} \frac{d u}{\rho(u)}\right) \int_{s}^{+\infty} \frac{d u}{\rho(u)}\right] f\left(s, x(s), y(s), x^{\prime}(s), y^{\prime}(s)\right) d s \\
& +\int_{\xi}^{t}\left[-\left((1-a) \int_{-\infty}^{+\infty} \frac{d u}{\rho(u)}-(1-a) b \int_{-\infty}^{\eta} \frac{d u}{\rho(u)}+a(1-b) \int_{-\infty}^{\xi} \frac{d u}{\rho(u)}\right) \int_{s}^{t} \frac{d u}{\rho(u)}\right. \\
& -\left((1-a) b \int_{-\infty}^{t} \frac{d u}{\rho(u)}+a b \int_{-\infty}^{\xi} \frac{d u}{\rho(u)}\right) \int_{s}^{\eta} \frac{d u}{\rho(u)} \\
& \left.+\left((1-a) \int_{-\infty}^{t} \frac{d u}{\rho(u)}+a \int_{-\infty}^{\xi} \frac{d u}{\rho(u)}\right) \int_{s}^{+\infty} \frac{d u}{\rho(u)}\right] f\left(s, x(s), y(s), x^{\prime}(s), y^{\prime}(s)\right) d s \\
& +\int_{t}^{\eta}\left[-\left((1-a) b \int_{-\infty}^{t} \frac{d u}{\rho(u)}+a b \int_{-\infty}^{\xi} \frac{d u}{\rho(u)}\right) \int_{s}^{\eta} \frac{d u}{\rho(u)}\right. \\
& \left.+\left((1-a) \int_{-\infty}^{t} \frac{d u}{\rho(u)}+a \int_{-\infty}^{\xi} \frac{d u}{\rho(u)}\right) \int_{s}^{+\infty} \frac{d u}{\rho(u)}\right] f\left(s, x(s), y(s), x^{\prime}(s), y^{\prime}(s)\right) d s \\
& +\int_{\eta}^{+\infty}\left[\left((1-a) \int_{-\infty}^{t} \frac{d u}{\rho(u)}+a \int_{-\infty}^{\xi} \frac{d u}{\rho(u)}\right) \int_{s}^{+\infty} \frac{d u}{\rho(u)}\right] f\left(s, x(s), y(s), x^{\prime}(s), y^{\prime}(s)\right) d s
\end{aligned}
$$


(3) $\xi<\eta \leq t$. We have

$$
\begin{aligned}
& x(t)=\int_{-\infty}^{\xi}\left[-\left[(1-a) \int_{-\infty}^{+\infty} \frac{d u}{\rho(u)}-(1-a) l \int_{-\infty}^{\eta} \frac{d u}{\rho(u)}+a(1-b) \int_{-\infty}^{\xi} \frac{d u}{\rho(u)}\right] \int_{s}^{t} \frac{d u}{\rho(u)}\right. \\
& +\left[(1-b) a \int_{-\infty}^{t} \frac{d u}{\rho(u)}-a \int_{-\infty}^{+\infty} \frac{d u}{\rho(u)}+a b \int_{-\infty}^{\eta} \frac{d u}{\rho(u)}\right] \int_{s}^{\xi} \frac{d u}{\rho(u)} \\
& -\left[(1-a) b \int_{-\infty}^{t} \frac{d u}{\rho(u)}+a b \int_{-\infty}^{\xi} \frac{d u}{\rho(u)}\right] \int_{s}^{\eta} \frac{d u}{\rho(u)} \\
& \left.+\left((1-a) \int_{-\infty}^{t} \frac{d u}{\rho(u)}+a \int_{-\infty}^{\xi} \frac{d u}{\rho(u)}\right) \int_{s}^{+\infty} \frac{d u}{\rho(u)}\right] f\left(s, x(s), y(s), x^{\prime}(s), y^{\prime}(s)\right) d s \\
& +\int_{\xi}^{\eta}\left[-\left((1-a) \int_{-\infty}^{+\infty} \frac{d u}{\rho(u)}-(1-a) b \int_{-\infty}^{\eta} \frac{d u}{\rho(u)}+a(1-b) \int_{-\infty}^{\xi} \frac{d u}{\rho(u)}\right) \int_{s}^{t} \frac{d u}{\rho(u)}\right. \\
& -\left((1-a) b \int_{-\infty}^{t} \frac{d u}{\rho(u)}+a b \int_{-\infty}^{\xi} \frac{d u}{\rho(u)}\right) \int_{s}^{\eta} \frac{d u}{\rho(u)} \\
& \left.+\left((1-a) \int_{-\infty}^{t} \frac{d u}{\rho(u)}+a \int_{-\infty}^{\xi} \frac{d u}{\rho(u)}\right) \int_{s}^{+\infty} \frac{d u}{\rho(u)}\right] f\left(s, x(s), y(s), x^{\prime}(s), y^{\prime}(s)\right) d s \\
& +\int_{\eta}^{t}\left[-\left[(1-a) \int_{-\infty}^{+\infty} \frac{d u}{\rho(u)}-(1-a) b \int_{-\infty}^{\eta} \frac{d u}{\rho(u)}+a(1-b) \int_{-\infty}^{\xi} \frac{d u}{\rho(u)}\right] \int_{s}^{t} \frac{d u}{\rho(u)}\right. \\
& \left.+\left((1-a) \int_{-\infty}^{t} \frac{d u}{\rho(u)}+a \int_{-\infty}^{\xi} \frac{d u}{\rho(u)}\right) \int_{s}^{+\infty} \frac{d u}{\rho(u)}\right] f\left(s, x(s), y(s), x^{\prime}(s), y^{\prime}(s)\right) d s \\
& +\int_{t}^{+\infty}\left[\left((1-a) \int_{-\infty}^{t} \frac{d u}{\rho(u)}+a \int_{-\infty}^{\xi} \frac{d u}{\rho(u)}\right) \int_{s}^{+\infty} \frac{d u}{\rho(u)}\right] f\left(s, x(s), y(s), x^{\prime}(s), y^{\prime}(s)\right) d s
\end{aligned}
$$

From Case (1), Case (2) and Case (3), we know that $x(t)=\int_{-\infty}^{+\infty} G(t, s) f\left(s, x(s), y(s), x^{\prime}(s), y^{\prime}(s)\right) d s$.

Similarly we can prove that $y(t)=\int_{-\infty}^{+\infty} H(t, s) g\left(s, x(s), y(s), x^{\prime}(s), y^{\prime}(s)\right) d s$. Then $(x, y)$ satisfies (2.9).

On the other hand, if $(x, y) \in E$ satisfies (2.9), it is easy to show that $(x, y)$ is a solution of $\operatorname{BVP}(1.5)$. The proof is completed.

Define the operator $T$ on $E$ by

$$
\begin{aligned}
& (T(x, y))(t)=\left(\left(T_{1}(x, y)\right)(t),\left(T_{2}(x, y)\right)(t)\right) \\
& =\left(\int_{-\infty}^{+\infty} G(t, s) f\left(s, x(s), y(s), x^{\prime}(s), y^{\prime}(s)\right) d s, \int_{-\infty}^{+\infty} H(t, s) g\left(s, x(s), y(s), x^{\prime}(s), y^{\prime}(s)\right) d s\right),(x, y) \in E .
\end{aligned}
$$

Remark 2.1. From (2.8.3), we know $\Delta>0$. Then $(1-a)^{2}+(1-b)^{2} \neq 0$. We suppose that $a \neq 1$. So for $(x, y) \in E$ satisfying

$$
\begin{aligned}
& \lim _{t \rightarrow-\infty} x(t)-a x(\xi)=0, \lim _{t \rightarrow+\infty} x(t)-b x(\eta)=0, \\
& \lim _{t \rightarrow-\infty} y(t)-c y(\xi)=0, \lim _{t \rightarrow+\infty} \varrho(t) y^{\prime}(t)-d \varrho(\eta) y^{\prime}(\eta)=0,
\end{aligned}
$$


we have that

$$
\begin{aligned}
& |x(t)| \leq\left|x(t)-\lim _{t \rightarrow-\infty} x(t)\right|+\left|\lim _{t \rightarrow-\infty} x(t)\right| \leq \int_{-\infty}^{t} \frac{1}{\rho(u)} \rho(u)\left|x^{\prime}(u)\right| d u+\frac{\left|\lim _{t \rightarrow-\infty} x(t)-a \lim _{t \rightarrow-\infty} x(t)\right|}{|1-a|} \\
& \leq \int_{-\infty}^{+\infty} \frac{d u}{\rho(u)} \sup _{t \in \mathbb{R}} \rho(t)\left|x^{\prime}(t)\right|+\frac{\left|a x(\xi)-a \lim _{t \rightarrow-\infty} x(t)\right|}{|1-a|} \leq \int_{-\infty}^{+\infty} \frac{d u}{\rho(u)} \sup _{t \in \mathbb{R}} \rho(t)\left|x^{\prime}(t)\right|+\frac{a\left|x(\xi)-\lim _{t \rightarrow-\infty} x(t)\right|}{|1-a|} \\
& \leq\left.\frac{|1-a|+a}{|1-a|} \int_{-\infty}^{+\infty} \frac{d u}{\rho(u)}|| \rho x^{\prime}\right|_{0} .
\end{aligned}
$$

It follows that $\|x\|_{0} \leq \frac{|1-a|+a}{|1-a|} \int_{-\infty}^{+\infty} \frac{d u}{\rho(u)}\left\|\rho x^{\prime}\right\|_{0}$.

Similarly we get from (2.8) that

$$
\begin{aligned}
& \|y\|_{0} \leq \frac{|1-c|+c}{|1-c|} \int_{-\infty}^{+\infty} \frac{d u}{\varrho(u)} \sup _{t \in R} \varrho(t)\left|y^{\prime}(t)\right| \\
& =\frac{1}{1-c} \int_{-\infty}^{+\infty} \frac{d u}{\varrho(u)}\left\|\varrho y^{\prime}\right\|_{0} .
\end{aligned}
$$

It follows that

$$
\begin{aligned}
& \|x\|_{X} \leq \max \left\{1, \frac{|1-a|+a}{|1-a|} \int_{-\infty}^{+\infty} \frac{d u}{\rho(u)}\right\}\left\|\rho x^{\prime}\right\|_{0}, \\
& \|y\|_{Y} \leq \max \left\{1, \frac{1}{1-c} \int_{-\infty}^{+\infty} \frac{d u}{\varrho(u)}\right\}\left\|\varrho y^{\prime}\right\|_{0} .
\end{aligned}
$$

Lemma 2.5. Suppose that (2.7), (2.8.1), (2.8.2), (2.8.3) and (2.8) hold. Then

(i) $(x, y) \in E$ is a positive solution of $\operatorname{BVP}(1.5)$ if and only if $(x, y)$ is a fixed point of $T$ in $X$.

(ii) $T: X \rightarrow X$ is well defined and is completely continuous.

Proof. (i) From Lemma 2.4, we know that $(x, y) \in E$ is a solution of $\operatorname{BVP}(1.5)$ if and only if $(x, y)$ is a fixed point of $T$ in $E$ i.e., $\left(T_{1}(x, y), T_{2}(x, y)\right)=(x, y)$.

Step 1. Prove that $\left(T_{1}(x, y)\right)(t)=x(t) \geq 0,\left(T_{2}(x, y)\right)=y(t) \geq 0$ for all $t \in \mathbb{R}$.

Note that $f, g$ are nonnegative Caratheodory functions. By the definition of $T_{1}, T_{2}$, it suffices to prove that $G(t, s) \geq 0$ and $H(t, s) \geq 0$ for all $t, s \in \mathbb{R}$. For ease expression, denote $\int_{a}^{b} \frac{d u}{\rho(u)}=\int_{a}^{b}$.

Case 1. For $s \geq \max \{\eta, t\}$, we see from (2.8.1) that

$$
\begin{aligned}
& \Delta G(t, s)=\left[(1-a) \int_{-\infty}^{t} \frac{d u}{\rho(u)}+a \int_{-\infty}^{\xi} \frac{d u}{\rho(u)}\right] \int_{s}^{+\infty} \frac{d u}{\rho(u)} \\
& \left\{\begin{array}{l}
=\left[(1-a) \int_{-\infty}^{t}+a \int_{-\infty}^{\xi}\right] \int_{s}^{+\infty} \geq 0, a \leq 1 \\
\geq\left[(1-a) \int_{-\infty}^{+\infty}+a \int_{-\infty}^{\xi}\right] \int_{s}^{+\infty} \geq 0, a>1 .
\end{array}\right.
\end{aligned}
$$


Case 2. For $s \leq \min \{\xi, t\}$, we have from (2.8.2) that

$$
\begin{aligned}
& \Delta G(t, s)=-\left[(1-a) \int_{-\infty}^{+\infty}-(1-a) b \int_{-\infty}^{\eta}+a(1-b) \int_{-\infty}^{\xi}\right] \int_{s}^{t} \\
& +\left[(1-b) a \int_{-\infty}^{t}-a \int_{-\infty}^{+\infty}+a b \int_{-\infty}^{\eta}\right] \int_{s}^{\xi} \\
& -\left[(1-a) b \int_{-\infty}^{t}+a b \int_{-\infty}^{\xi}\right] \int_{s}^{\eta}+\left[(1-a) \int_{-\infty}^{t}+a \int_{-\infty}^{\xi}\right] \int_{s}^{+\infty} \\
& =\int_{-\infty}^{s}\left[\int_{\eta}^{+\infty}+(1-b) \int_{t}^{\eta}\right] \\
& \left\{\begin{array}{l}
=\int_{-\infty}^{s}\left[\int_{\eta}^{+\infty}+(1-b) \int_{t}^{\eta}\right] \geq 0, b \leq 1, \\
\geq \int_{-\infty}^{s}\left[\int_{\eta}^{+\infty}+(1-b) \int_{-\infty}^{\eta}\right] \geq 0, b>1 .
\end{array}\right.
\end{aligned}
$$

Case 3. For $t<s \leq \xi<\eta$, we have from (2.8.2) that

$$
\begin{aligned}
& \Delta G(t, s)=\left[(1-b) a \int_{-\infty}^{t}-a \int_{-\infty}^{+\infty}+a b \int_{-\infty}^{\eta}\right] \int_{s}^{\xi}-\left[(1-a) b \int_{-\infty}^{t}+a b \int_{-\infty}^{\xi}\right] \int_{s}^{\eta} \\
& +\left[(1-a) \int_{-\infty}^{t}+a \int_{-\infty}^{\xi}\right] \int_{s}^{+\infty} \\
& =\int_{-\infty}^{t}\left(\int_{\eta}^{+\infty}+(1-b) \int_{s}^{\eta}\right)+a \int_{t}^{s}\left(\int_{\eta}^{+\infty}+(1-b) \int_{\xi}^{\eta}\right) \geq 0 .
\end{aligned}
$$

Case 4. For $\max \{t, \xi\}<s \leq \eta$, we have from (2.8.1) and (2.8.2) that

$$
\begin{aligned}
& \Delta G(t, s)=-\left[(1-a) b \int_{-\infty}^{t}+a b \int_{-\infty}^{\xi}\right] \int_{s}^{\eta}+\left[(1-a) \int_{-\infty}^{t}+a \int_{-\infty}^{\xi}\right] \int_{s}^{+\infty} \\
& \geq\left(\int_{s}^{+\infty}-b \int_{s}^{\eta}\right)\left(\int_{-\infty}^{t}-a \int_{\xi}^{t}\right) \\
& =\left(\int_{\eta}^{+\infty}+(1-b) \int_{s}^{\eta}\right)\left(\int_{-\infty}^{\xi}+(1-a) \int_{\xi}^{t}\right) \\
& \left\{\begin{array}{l}
\geq 0, a \leq 1, b \leq 1, \\
\geq\left(\int_{\eta}^{+\infty}+(1-b) \int_{-\infty}^{\eta}\right)\left(\int_{-\infty}^{\xi}+(1-a) \int_{\xi}^{t}\right) \geq 0, a>1, b \leq 1, \\
\geq\left(\int_{\eta}^{+\infty}+(1-b) \int_{s}^{\eta}\right)\left(\int_{-\infty}^{\xi}+(1-a) \int_{\xi}^{+\infty}\right) \geq 0, a \leq 1, b>1, \\
\geq\left(\int_{\eta}^{+\infty}+(1-b) \int_{-\infty}^{\eta}\right)\left(\int_{-\infty}^{\xi}+(1-a) \int_{\xi}^{+\infty}\right), a>1, b>1 .
\end{array}\right.
\end{aligned}
$$


Case 5. For $\xi<s \leq \min \{t, \eta\}$, we have from (2.8.1) and (2.8.2) that

$$
\begin{aligned}
& \Delta G(t, s)=-\left[(1-a) \int_{-\infty}^{+\infty}-(1-a) b \int_{-\infty}^{\eta}+a(1-b) \int_{-\infty}^{\xi}\right] \int_{s}^{t} \\
& -\left[(1-a) b \int_{-\infty}^{t}+a b \int_{-\infty}^{\xi}\right] \int_{s}^{\eta}+\left[(1-a) \int_{-\infty}^{t}+a \int_{-\infty}^{\xi}\right] \int_{s}^{+\infty} \\
& \left\{\begin{array}{l}
=\int_{t}^{+\infty}\left((1-a) \int_{-\infty}^{s}+a \int_{-\infty}^{\xi}\right)+b \int_{\eta}^{t}\left((1-a) \int_{-\infty}^{s}+a \int_{-\infty}^{\xi}\right) \geq 0, \eta \leq t, \\
=\left((1-a) \int_{-\infty}^{s}+a \int_{-\infty}^{\xi}\right)\left(\int_{\eta}^{+\infty}+(1-b) \int_{t}^{\eta}\right) \geq 0, \eta>t .
\end{array}\right.
\end{aligned}
$$

Case 6. For $\xi<\eta<s \leq t$, we have (2.8.1) that

$$
\begin{aligned}
& \Delta G(t, s)=-\left[(1-a) \int_{-\infty}^{+\infty}-(1-a) b \int_{-\infty}^{\eta}+a(1-b) \int_{-\infty}^{\xi}\right] \int_{s}^{t} \\
& +\left[(1-a) \int_{-\infty}^{t}+a \int_{-\infty}^{\xi}\right] \int_{s}^{+\infty} \\
& =\int_{t}^{+\infty}\left((1-a) \int_{-\infty}^{s}+a \int_{-\infty}^{\xi}\right)+b \int_{s}^{t}\left((1-a) \int_{-\infty}^{\eta}+a \int_{-\infty}^{\xi}\right) \geq 0 .
\end{aligned}
$$

From cases 1-6 and (2.8.3), we have $G(t, s) \geq 0$. Similarly we can prove that $H(t, s) \geq 0$.

Step 2. We prove that $x(t)>0$ and $y(t)>0$ on $R$ if $(x, y)$ is a solution of $\operatorname{BVP}(1.5)$.

Suppose that there exists $t_{0} \in \mathbb{R}$ such that $x\left(t_{0}\right)=0$. Since $\left(\rho(t) x^{\prime}(t)\right)^{\prime}=-f(t, x(t), y(t)$, $\left.x^{\prime}(t), y^{\prime}(t)\right) \leq 0$ for all $t \in \mathbb{R}$, we know that $\rho(t) x^{\prime}(t) \geq 0$ on $R$ or $\rho(t) x^{\prime}(t) \leq 0$ on $\mathbb{R}$ or there exists $t_{1} \in R$ such that $\rho(t) x^{\prime}(t) \geq 0$ on $\left(-\infty, t_{1}\right]$ and $\rho(t) x^{\prime}(t) \leq 0$ on $\left[t_{1},+\infty\right)$. We consider three cases:

Case 1. $\rho(t) x^{\prime}(t) \leq 0$ for all $t \in \mathbb{R}$. Then $x^{\prime}(t) \leq 0$ for all $t \in \mathbb{R}$. It is easy to see that $x(t) \equiv 0$ for all $t \in\left[t_{0},+\infty\right)$. Then $f\left(t, 0, y(t), 0, y^{\prime}(t)\right)=-\left(\rho(t) x^{\prime}(t)\right)^{\prime} \equiv 0$ on $\left[t_{0},+\infty\right)$, a contradiction.

Case 2. $\rho(t) x^{\prime}(t) \geq 0$ for all $t \in \mathbb{R}$. Then $x^{\prime}(t) \geq 0$ on $\mathbb{R}$. It is easy to see that $x(t) \equiv 0$ for all $t \in\left(-\infty, t_{0}\right]$. Then $f\left(t, 0, y(t), 0, y^{\prime}(t)\right)=-\left(\rho(t) x^{\prime}(t)\right)^{\prime} \equiv 0$ on $\left(-\infty, t_{0}\right]$, a contradiction.

Case 3. there exists $t_{1} \in \mathbb{R}$ such that $\rho(t) x^{\prime}(t) \geq 0$ for $t \in\left(-\infty, t_{1}\right]$ and $\rho(t) x^{\prime}(t) \leq 0$ for $t \in\left[t_{1},+\infty\right)$. Then $x^{\prime}(t) \geq 0$ on $\left(-\infty, t_{1}\right]$ and $x^{\prime}(t) \leq 0$ on $\left[t_{1},+\infty\right)$. When $t_{0} \leq t_{1}$, we get $x(t) \equiv 0$ for all $t \in\left(-\infty, t_{0}\right]$, thus $f\left(t, 0, y(t), 0, y^{\prime}(t)\right)=-\left(\rho(t) x^{\prime}(t)\right)^{\prime} \equiv 0$ on $\left(-\infty, t_{0}\right]$, a contradiction. When $t_{0} \geq t_{1}$, we get $x(t) \equiv 0$ for all $t \in\left[t_{0},+\infty\right)$. Then $f\left(t, 0, y(t), 0, y^{\prime}(t)\right)=-\left(\rho(t) x^{\prime}(t)\right)^{\prime} \equiv 0$ on $\left[t_{0},+\infty\right)$, a contradiction.

From above discussion, we know that $x$ is positive on $\mathbb{R}$. Similarly we can prove that $y$ is positive on $\mathbb{R}$. We obtain $(x, y)$ is a positive solution of $\operatorname{BVP}(1.5)$. This completes the proof of (i).

(ii) We prove that $T$ is completely continuous.

Step 1. we prove that $T:(x, y)=\left(T_{1}(x, y), T_{2}(x, y)\right)$ is continuous on $E$. It suffices to prove that both $T_{1}:(x, y) \rightarrow T_{1}(x, y)$ and $T_{2}:(x, y) \rightarrow T_{2}(x, y)$ are continuous. Since $f, g$ are Carathéodory functions, the result follows.

Step 2. we show that $T$ is maps bounded subsets into bounded sets.

Given a bounded set $D \subseteq E$. Then, there exists $M>0$ such that $D \subseteq\{(x, y) \in E:\|x\|,\|y\| \leq$ 
$M\}$. Then there exists $\varphi_{M} \in L^{1}(R)$ such that

$$
\begin{aligned}
& \left|f\left(t, x(t), y(t), x^{\prime}(t), y^{\prime}(t)\right)\right| \leq \varphi_{r}(t), \text { a.e. }, t \in \mathbb{R}, \\
& \left|g\left(t, x(t), y(t), x^{\prime}(t), y^{\prime}(t)\right)\right| \leq \varphi_{r}(t), \text { a.e., } t \in \mathbb{R} .
\end{aligned}
$$

By the definitions of $T_{1}$ and $T_{2}$, we have

$$
\left\{\begin{array}{l}
{\left[\rho(t)\left(T_{1}(x, y)\right)^{\prime}(t)\right]^{\prime}+f\left(t, x(t), y(t), x^{\prime}(t), y^{\prime}(t)\right)=0, a . e ., t \in \mathbb{R},} \\
{\left[\varrho(t)\left(T_{2}(x, y)\right)^{\prime}(t)\right]^{\prime}+g\left(t, x(t), y(t), x^{\prime}(t), y^{\prime}(t)\right)=0, a . e ., t \in \mathbb{R},} \\
\lim _{t \rightarrow-\infty}\left(T_{1}(x, y)\right)(t)-a\left(T_{1}(x, y)\right)(\xi)=0, \lim _{t \rightarrow+\infty}\left(T_{1}(x, y)\right)(t)-b\left(T_{1}(x, y)\right)(\eta)=0, \\
\lim _{t \rightarrow-\infty}\left(T_{2}(x, y)\right)(t)-c\left(T_{2}(x, y)\right)(\xi)=0, \lim _{t \rightarrow+\infty} \varrho(t)\left(T_{2}(x, y)\right)^{\prime}(t)-d \varrho(\eta)\left(T_{2}(x, y)\right)^{\prime}(\eta)=0 .
\end{array}\right.
$$

By the definitions of $T_{1}$ and $T_{2}$, we have

$$
\begin{aligned}
& \rho(t)\left(T_{1}(x, y)\right)^{\prime}(t)=\frac{1}{\Delta}\left[-(1-a) b \int_{-\infty}^{\eta}\left(\int_{s}^{\eta} \frac{d u}{\rho(u)}\right) f\left(s, x(s), y(s), x^{\prime}(s), y^{\prime}(s)\right) d s\right. \\
& +(1-a) \int_{-\infty}^{+\infty}\left(\int_{s}^{+\infty} \frac{d u}{\rho(u)}\right) f\left(s, x(s), y(s), x^{\prime}(s), y^{\prime}(s)\right) d s \\
& \left.+(1-b) a \int_{-\infty}^{\xi}\left(\int_{s}^{\xi} \frac{d u}{\rho(u)}\right) f\left(s, x(s), y(s), x^{\prime}(s), y^{\prime}(s)\right) d s\right]-\int_{-\infty}^{t} f\left(s, x(s), y(s), x^{\prime}(s), y^{\prime}(s)\right) d s, \\
& \varrho(t)\left(T_{2}(x, y)\right)^{\prime}(t)=-\frac{d}{1-d} \int_{-\infty}^{\eta} f\left(s, x(s), y(s), x^{\prime}(s), y^{\prime}(s)\right) d s \\
& +\frac{1}{1-d} \int_{-\infty}^{+\infty} f\left(s, x(s), y(s), x^{\prime}(s), y^{\prime}(s)\right) d s-\int_{-\infty}^{t} f\left(s, x(s), y(s), x^{\prime}(s), y^{\prime}(s)\right) d s .
\end{aligned}
$$

So

$$
\begin{aligned}
& \rho(t)\left|\left(T_{1}(x, y)\right)^{\prime}(t)\right| \leq \frac{1}{\Delta}\left[|1-a| b \int_{-\infty}^{+\infty} \frac{d u}{\rho(u)}+|1-a| \int_{-\infty}^{+\infty} \frac{d u}{\rho(u)}\right. \\
& \left.+|1-b| a \int_{-\infty}^{+\infty} \frac{d u}{\rho(u)}+\Delta\right] \int_{-\infty}^{+\infty} \varphi_{r}(s) d s \\
& \varrho(t)\left|\left(T_{2}(x, y)\right)^{\prime}(t)\right| \leq\left(\frac{1+d}{1-d}+1\right) \int_{-\infty}^{+\infty} \varphi_{r}(s) d s .
\end{aligned}
$$


Similarly to the Remark 2.1, we have

$$
\begin{aligned}
& || T_{1}(x, y) \|_{X}=\max \left\{\sum_{t \in R}\left|\left(T_{1}(x, y)\right)(t), \sup _{t \in R} \rho(t)\right|\left(T_{1}(x, y)\right)^{\prime}(t) \mid\right\} \\
& \leq \max \left\{1, \frac{|1-a|+a}{|1-a|} \int_{-\infty}^{+\infty} \frac{d u}{\rho(u)}\right\} \sup _{t \in R} \rho(t)\left|\left(T_{1}(x, y)\right)^{\prime}(t)\right| \\
& \leq \max \left\{1, \frac{|1-a|+a}{|1-a|} \int_{-\infty}^{+\infty} \frac{d u}{\rho(u)}\right\} \frac{1}{\Delta}\left[|1-a| b \int_{-\infty}^{+\infty} \frac{d u}{\rho(u)}+|1-a| \int_{-\infty}^{+\infty} \frac{d u}{\rho(u)}\right. \\
& \left.+|1-b| a \int_{-\infty}^{+\infty} \frac{d u}{\rho(u)}+\Delta\right] \int_{-\infty}^{+\infty} \varphi_{r}(s) d s
\end{aligned}
$$

and

$$
\begin{aligned}
& || T_{2}(x, y) \|_{Y}=\max \left\{\sum_{t \in R}\left|\left(T_{2}(x, y)\right)(t), \sup _{t \in R} \varrho(t)\right|\left(T_{2}(x, y)\right)^{\prime}(t) \mid\right\} \\
& \leq \max \left\{1, \frac{1}{1-c} \int_{-\infty}^{+\infty} \frac{d u}{\varrho(u)}\right\} \sup _{t \in R} \rho(t)\left|\left(T_{2}(x, y)\right)^{\prime}(t)\right| \int_{-\infty}^{+\infty} \varphi_{r}(s) d s \\
& \leq \max \left\{1, \frac{1}{1-c} \int_{-\infty}^{+\infty} \frac{d u}{\varrho(u)}\right\}\left(\frac{1+d}{1-d}+1\right) \int_{-\infty}^{+\infty} \varphi_{r}(s) d s .
\end{aligned}
$$

It follows that

$$
\|(T(x, y))\|=\max \left\{\left\|T_{1}(x, y)\right\|_{X},\left\|T_{2}(x, y)\right\|_{Y}\right\}<\infty .
$$

So, $\{T D\}$ is uniformly bounded.

Step 3. we prove that both $\left\{t \rightarrow\left(T_{1}(x, y)\right)(t):(x, y) \in D\right\},\left\{t \rightarrow\left(T_{2}(x, y)\right)(t):(x, y) \in D\right\}$ and $\left\{t \rightarrow\left(\rho(t)\left(T_{1}(x, y)\right)^{\prime}(t):(x, y) \in D\right\}\right.$ and $\left\{t \rightarrow\left(\rho(t)\left(T_{2}(x, y)\right)^{\prime}(t):(x, y) \in D\right\}\right.$ are equi-continuous on each finite subinterval on $\mathbb{R}$.

The proof is standard and is omitted. One may see [19].

Step 4. we show that both $\left\{t \rightarrow\left(T_{1}(x, y)\right)(t):(x, y) \in D\right\},\left\{t \rightarrow\left(T_{2}(x, y)\right)(t):(x, y) \in D\right\}$ and $\left\{t \rightarrow\left(\rho(t)\left(T_{1}(x, y)\right)^{\prime}(t):(x, y) \in D\right\}\right.$ and $\left\{t \rightarrow\left(\rho(t)\left(T_{2}(x, y)\right)^{\prime}(t):(x, y) \in D\right\}\right.$ are equi-convergent at both $+\infty$ and $-\infty$ respectively.

We have that

$$
\begin{aligned}
& \mid\left(T_{1}(x, y)\right)(t)-\left[-\frac{a}{\Delta} \int_{-\infty}^{+\infty} \frac{d u}{\rho(u)}+\frac{a b}{\Delta} \int_{-\infty}^{\eta} \frac{d u}{\rho(u)}\right] \int_{-\infty}^{\xi}\left(\int_{s}^{\xi} \frac{d u}{\rho(u)}\right) f\left(s, x(s), y(s), x^{\prime}(s), y^{\prime}(s)\right) d s \\
& -\left[\frac{a b}{\Delta} \int_{-\infty}^{\xi} \frac{d u}{\rho(u)}\right] \int_{-\infty}^{\eta}\left(\int_{s}^{\eta} \frac{d u}{\rho(u)}\right) f\left(s, x(s), y(s), x^{\prime}(s), y^{\prime}(s)\right) d s \\
& +\left[\frac{a}{\Delta} \int_{-\infty}^{\xi} \frac{d u}{\rho(u)}\right] \int_{-\infty}^{+\infty}\left(\int_{s}^{+\infty} \frac{d u}{\rho(u)}\right) f\left(s, x(s), y(s), x^{\prime}(s), y^{\prime}(s)\right) d s \mid \\
& \leq \int_{-\infty}^{t} \varphi_{r}(s) d s \int_{-\infty}^{+\infty} \frac{d u}{\rho(u)}+\left[\frac{|1-b| a}{\Delta}+\frac{|1-a| b}{\Delta}+\frac{|1-a|}{\Delta}\right] \int_{-\infty}^{t} \frac{d u}{\rho(u)} \int_{-\infty}^{+\infty} \varphi_{r}(s) d s \int_{-\infty}^{+\infty} \frac{d u}{\rho(u)}
\end{aligned}
$$

$\rightarrow 0$ uniformly as $t \rightarrow-\infty$. 
Further more, we have that

$$
\begin{aligned}
& \mid \rho(t)\left(T_{1}(x, y)\right)^{\prime}(t)-\frac{1}{\Delta}\left[-(1-a) b \int_{-\infty}^{\eta}\left(\int_{s}^{\eta} \frac{d u}{\rho(u)}\right) f\left(s, x(s), y(s), x^{\prime}(s), y^{\prime}(s)\right) d s\right. \\
& +(1-a) \int_{-\infty}^{+\infty}\left(\int_{s}^{+\infty} \frac{d u}{\rho(u)}\right) f\left(s, x(s), y(s), x^{\prime}(s), y^{\prime}(s)\right) d s \\
& \left.+(1-b) a \int_{-\infty}^{\xi}\left(\int_{s}^{\xi} \frac{d u}{\rho(u)}\right) f\left(s, x(s), y(s), x^{\prime}(s), y^{\prime}(s)\right) d s\right] \mid \\
& \leq \int_{-\infty}^{t} \varphi_{r}(s) d s \rightarrow 0 \text { uniformly as } t \rightarrow-\infty .
\end{aligned}
$$

Hence $\left\{t \rightarrow \rho(t)\left(T_{1}(x, y)\right)^{\prime}(t):(x, y) \in D\right\}$ and $\left\{t \rightarrow\left(T_{1}(x, y)\right)(t):(x, y) \in D\right\}$ are equiconvergent as $t \rightarrow-\infty$. Similarly we can prove that $\left\{t \rightarrow \rho(t)\left(T_{1}(x, y)\right)^{\prime}(t):(x, y) \in D\right\}$ and $\left\{t \rightarrow\left(T_{1}(x, y)\right)(t):(x, y) \in D\right\}$ are equiconvergent as $t \rightarrow+\infty$.

Similarly we can prove that both $\left\{t \rightarrow\left(T_{2}(x, y)\right)(t):(x, y) \in D\right\}$ and $\left\{t \rightarrow\left(\rho(t)\left(T_{2}(x, y)\right)^{\prime}(t):\right.\right.$ $(x, y) \in D\}$ are equiconvergent as $t \rightarrow \pm \infty$. The details are omitted.

From Steps 1-4, we see that $T$ maps bounded sets into relatively compact sets.

Therefore, the operator $T: E \rightarrow E$ is completely continuous. The proof of (ii) is complete. Thus the proof of Lemma 2.5 is ended.

\section{Main results}

Now, we prove the main theorems in this paper by using the Schauder's fixed point theorem. We need the following assumptions:

(D1) $f, g$ are $(\rho, \varrho)$-Carathéodory functions and satisfy that there exist non-decreasing functions $\Phi, \Psi: \mathbb{R}^{+^{4}} \rightarrow \mathbb{R}^{+}$, measurable functions $\varphi, \psi, \varphi_{1}, \psi_{1}$ such that

$$
\begin{aligned}
& \left|f\left(t, x, y, \frac{u}{\rho(t)}, \frac{v}{\varrho(t)}\right)-\varphi(t)\right| \leq \varphi_{1}(t) \Phi(x, y, u, v), x, y, u, v \in \mathbb{R}, \text { a.e., } t \in \mathbb{R}, \\
& \left|g\left(t, x, y, \frac{u}{\rho(t)}, \frac{v}{\varrho(t)}\right)-\psi(t)\right| \leq \psi_{1}(t) \Psi(x, y, u, v), x, y, u, v \in \mathbb{R}, a . e ., t \in \mathbb{R} .
\end{aligned}
$$

Define

Denote

$$
\bar{\Phi}(t)=\int_{-\infty}^{+\infty} G(t, s) \varphi(s) d s, \bar{\Psi}(t)=\int_{-\infty}^{+\infty} H(t, s) \psi(s) d s
$$

$$
\begin{aligned}
& P=\max \left\{1, \frac{|1-a|+a}{|1-a|} \int_{-\infty}^{+\infty} \frac{d u}{\rho(u)}\right\} \frac{1}{\Delta}\left[|1-a| b \int_{-\infty}^{+\infty} \frac{d u}{\rho(u)}+|1-a| \int_{-\infty}^{+\infty} \frac{d u}{\rho(u)}\right. \\
& \left.+|1-b| a \int_{-\infty}^{+\infty} \frac{d u}{\rho(u)}+\Delta\right]\left\|\varphi_{1}\right\|_{1}, \\
& Q=\max \left\{1, \frac{1}{1-c} \int_{-\infty}^{+\infty} \frac{d u}{\varrho(u)}\right\}\left(\frac{1+d}{1-d}+1\right)\left\|\psi_{1}\right\|_{1} .
\end{aligned}
$$

Theorem 3.1. Suppose that (2.7), (2.8.1), (2.8.2), (2.8.3) and (2.8), (D1) hold. Then BVP(1.5) has at least one positive solution if

$$
\begin{aligned}
& \left.P \Phi\left(r_{1}+\|\bar{\Phi}\|, r_{2}+\|\bar{\Psi}\|\right), r_{1}+\|\bar{\Phi}\|, r_{2}+\|\bar{\Psi}\|\right) \leq r_{1}, \\
& \left.\left.Q \Psi\left(r_{1}+\|\bar{\Phi}\|, r_{2}+\|\bar{\Psi}\|\right), r_{1}+\|\bar{\Phi}\|, r_{2}+\|\bar{\Psi}\|\right)\right) \leq r_{2} .
\end{aligned}
$$


has a couple of positive solution $\left(r_{1}, r_{2}\right)$.

Proof. To apply the Schauder's fixed point theorem, we should define an closed convex bounded subset $\Omega$ of $E$ such that $T(\Omega) \subseteq \Omega$. By the definitions of $\bar{\Phi}, \bar{\Psi}$, we have $(\bar{\Phi}, \bar{\Psi}) \in E$ and

$$
\left\{\begin{array}{l}
{\left[\rho(t) \bar{\Phi}^{\prime}(t)\right]^{\prime}+\varphi(t)=0, \text { a.e., } t \in R} \\
{\left[\varrho(t) \bar{\Psi}^{\prime}(t)\right]^{\prime}+\psi(t)=0, \text { a.e., } t \in R} \\
\lim _{t \rightarrow-\infty} \bar{\Phi}(t)-a \bar{\Phi}(\xi)=0, \lim _{t \rightarrow+\infty} \bar{\Phi}(t)-b \bar{\Phi}(\eta)=0 \\
\lim _{t \rightarrow-\infty} \bar{\Psi}(t)-c \bar{\Psi}(\xi)=0, \lim _{t \rightarrow+\infty} \varrho(t) \bar{\Psi}^{\prime}(t)-d \varrho(\eta) \bar{\Psi}^{\prime}(\eta)=0
\end{array}\right.
$$

Let $r_{1}>0, r_{2}>0$, denote $\Omega=\left\{(x, y) \in E:\|x-\bar{\Phi}\| \leq r_{1},\|y-\bar{\Psi}\| \leq r_{2}\right\}$. For $(x, y) \in \Omega$, we get

$$
\|x\| \leq\|x-\bar{\Phi}\|+\|\bar{\Phi}\| \leq r_{1}+\|\bar{\Phi}\|, \quad\|y\| \leq\|y-\bar{\Psi}\|+\|\bar{\Psi}\| \leq r_{2}+\|\bar{\Psi}\| .
$$

Then (D1) implies that

$$
\begin{aligned}
& \left|f_{1}\left(t, x(t), y(t), x^{\prime}(t), y^{\prime}(t)\right)-\varphi(t)\right|=\left|f\left(t, x(t), y(t), \frac{\rho(t) x^{\prime}(t)}{\rho(t)}, \frac{\varrho(t) y^{\prime}(t)}{\varrho(t)}\right)-\varphi(t)\right| \\
& \leq \varphi_{1}(t) \Phi(\|x\|,\|y\|,\|x\|,\|y\|) \\
& \leq \varphi_{1}(t) \Phi\left(r_{1}+\|\bar{\Phi}\|, r_{2}+\|\bar{\Psi}\|, r_{1}+\|\bar{\Phi}\|, r_{2}+\|\bar{\Psi}\|\right), \text { a.e., } t \in R, \\
& \left|g\left(t, x(t), y(t), x^{\prime}(t), y^{\prime}(t)\right)-\psi(t)\right| \leq \psi_{1}(t) \Psi\left(r_{1}+\|\bar{\Phi}\|, r_{2}+\|\bar{\Psi}\|, r_{1}+\|\bar{\Phi}\|, r_{2}+\|\bar{\Psi}\|\right), \text { a.e., } t \in R .
\end{aligned}
$$

By the definition of $T$ and Lemma 2.5, we have $\left(T_{1}(x, y), T_{2}(x, y)\right) \in P$. By the methods used in (2.11), we get that

$$
\begin{aligned}
& \left\|\left(T_{1}(x, y)\right)-\bar{\Phi}\right\| \leq \max \left\{1, \frac{|1-a|+a}{|1-a|} \int_{-\infty}^{+\infty} \frac{d u}{\rho(u)}\right\} \sup _{t \in R} \rho(t)\left|\left[\left(T_{1}(x, y)\right)(t)-\bar{\Phi}(t)\right]^{\prime}\right| \\
& \leq \max \left\{1, \frac{|1-a|+a}{|1-a|} \int_{-\infty}^{+\infty} \frac{d u}{\rho(u)}\right\} \frac{1}{\Delta}\left[|1-a| b \int_{-\infty}^{+\infty} \frac{d u}{\rho(u)}+|1-a| \int_{-\infty}^{+\infty} \frac{d u}{\rho(u)}\right. \\
& \left.+|1-b| a \int_{-\infty}^{+\infty} \frac{d u}{\rho(u)}+\Delta\right] \int_{-\infty}^{+\infty} \varphi_{1}(s) d s \Phi\left(r_{1}+\|\bar{\Phi}\|, r_{2}+\|\bar{\Psi}\|, r_{1}+\|\bar{\Phi}\|, r_{2}+\|\bar{\Psi}\|\right) \\
& =P \Phi\left(r_{1}+\|\Phi\|, r_{2}+\|\Psi\|, r_{1}+\|\Phi\|, r_{2}+\|\Psi\|\right) .
\end{aligned}
$$

Similarly, we have

$$
\left\|T_{2}(x, y)-\bar{\Psi}\right\| \leq Q \Psi\left(r_{1}+\|\Phi\|, r_{2}+\|\Psi\|, r_{1}+\|\Phi\|, r_{2}+\|\Psi\|\right) .
$$

From the assumption, the inequality system (3.12) has positive solution $\left(r_{1}, r_{2}\right)$. We choose $\Omega=\left\{(x, y) \in E:\|x-\bar{\Phi}\| \leq r_{1},\|y-\bar{\Psi}\| \leq r_{2}\right\}$. Then we get $T(\Omega) \subset \Omega$. Hence the Schauder's 
fixed point theorem implies that $T$ has a fixed point $(x, y) \in \Omega$. Lemma 2.5 imply that $(x, y)$ is a positive solution of $\operatorname{BVP}(1.5)$. The proof of Theorem 3.1 is complete.

(D2) $f, g$ are Carathéodory functions and satisfy that there exist nonnegative constants $b_{i}, a_{i}$, $\tau_{j}, \sigma_{j}, \delta_{j}, \gamma_{j}(j=1,2, \cdots, n)$ measurable functions $\varphi, \psi: \mathbb{R} \rightarrow \mathbb{R}$ such that

$$
\begin{gathered}
\left|f\left(t, x, y, \frac{u}{\rho(t)}, \frac{v}{\varrho(t)}\right)-\varphi(t)\right| \leq \varphi_{1}(t) \sum_{j=1}^{n} a_{j}|x|^{\tau_{j}}|y|^{\sigma_{j}}|u|^{\delta_{j}}|v|^{\gamma_{j}}, t \in \mathbb{R}, x, y, u, v \in \mathbb{R}, \\
\left|g\left(t, x, y, \frac{u}{\rho(t)}, \frac{v}{\varrho(t)}\right)-\psi(t)\right| \leq \psi_{1}(t) \sum_{j=1}^{n} b_{j}|x|^{\tau_{j}}|y|^{\sigma_{j}}|u|^{\delta_{j}}|v|^{\gamma_{j}}, t \in \mathbb{R}, x, y, u, v \in \mathbb{R} .
\end{gathered}
$$

Define

$$
\bar{\Phi}(t)=\int_{-\infty}^{+\infty} G(t, s) \varphi(s) d s, \bar{\Psi}(t)=\int_{-\infty}^{+\infty} H(t, s) \psi(s) d s
$$

Denote

$$
\begin{aligned}
& A \geq \max \{\|\bar{\Phi}\|,\|\bar{\Psi}\|\} \\
& \sigma=\max \left\{\tau_{j}+\sigma_{j}+\delta_{j}+\gamma_{j}: j=1,2, \cdots, n\right\} \\
& M=\max \left\{\operatorname { m a x } \{ 1 , \frac { | 1 - a | + a } { | 1 - a | } \int _ { - \infty } ^ { + \infty } \frac { d u } { \rho ( u ) } \} \left[\frac{|1-a| b}{\Delta} \int_{-\infty}^{+\infty} \frac{d u}{\rho(u)}+\frac{|1-a|}{\Delta} \int_{-\infty}^{+\infty} \frac{d u}{\rho(u)}\right.\right. \\
& \left.+\frac{|1-b| a}{\Delta} \int_{-\infty}^{+\infty} \frac{d u}{\rho(u)}+1\right]\left\|\varphi_{1}\right\|_{1} \sum_{j=1}^{n} a_{j} A^{\tau_{j}+\delta_{j}+\sigma_{j}+\gamma_{j}-\sigma}, \\
& \left.\quad \max \left\{1, \frac{1}{1-c} \int_{-\infty}^{+\infty} \frac{d u}{\varrho(u)}\right\}\left(\frac{1+d}{1-d}+1\right)\left\|\psi_{1}\right\|_{1} \sum_{j=1}^{n} b_{j} A^{\tau_{j}+\delta_{j}+\sigma_{j}+\gamma_{j}-\sigma}\right\}
\end{aligned}
$$

Theorem 3.2. Suppose that (2.7), (2.8.1), (2.8.2), (2.8.3) and (2.8), (D2) hold. Then BVP(1.5) has at least one positive solution if

$$
\sigma \in[0,1) \text { or } \sigma=1 \text { with } M<1 \text { or } \sigma>1 \text { with } \frac{\sigma^{\sigma} A^{\sigma-1}}{(\sigma-1)^{\sigma-1}} \leq \frac{1}{M} .
$$

Proof: To apply the Schauder's fixed point theorem, we should define an closed convex bounded subset $\Omega$ of $E$ such that $T(\Omega) \subseteq \Omega$.

Let $r>0$, denote $\Omega=\{(x, y) \in E:\|(x, y)-(\bar{\Phi}, \bar{\Psi})\| \leq r\}$. For $(x, y) \in \Omega$, we get $\|x\| \leq$ $\|x-\bar{\Phi}\|+\|\bar{\Phi}\| \leq r+\|\bar{\Phi}\|, \quad\|y\| \leq\|y-\bar{\Psi}\|+\|\bar{\Psi}\| \leq r+\|\bar{\Psi}\|$. Then (D2) implies that

$$
\begin{aligned}
& \left|f\left(t, x(t), y(t), x^{\prime}(t), y^{\prime}(t)\right)-\varphi(t)\right| \leq \varphi_{1}(t) \sum_{j=1}^{n} a_{j}[r+\| \bar{\Phi}||]^{\tau_{j}+\delta_{j}}[r+\| \bar{\Psi}||]^{\sigma_{j}+\gamma_{j}}, t \in \mathbb{R}, \\
& \left|g\left(t, x(t), y(t), x^{\prime}(t), y^{\prime}(t)\right)-\psi(t)\right| \leq \psi_{1}(t) \sum_{j=1}^{n} b_{j}[r+\| \bar{\Phi}||]^{\tau_{j}+\delta_{j}}[r+\|\bar{\Psi}\|]^{\sigma_{j}+\gamma_{j}}, t \in \mathbb{R} .
\end{aligned}
$$


By the definition of $T$, using above inequalities and (2.11), we get that

$$
\begin{aligned}
& || T_{1}(x, y)-\bar{\Phi}|| \leq \max \left\{1, \frac{|1-a|+a}{|1-a|} \int_{-\infty}^{+\infty} \frac{d u}{\rho(u)}\right\} \sup _{t \in \mathbb{R}} \rho(t)\left|\left(T_{1}(x, y)\right)^{\prime}(t)-\bar{\Phi}^{\prime}(t)\right| \\
& \leq \max \left\{1, \frac{|1-a|+a}{|1-a|} \int_{-\infty}^{+\infty} \frac{d u}{\rho(u)}\right\}\left[\frac{|1-a| b}{\Delta} \int_{-\infty}^{+\infty} \frac{d u}{\rho(u)}+\frac{|1-a|}{\Delta} \int_{-\infty}^{+\infty} \frac{d u}{\rho(u)}\right. \\
& \left.+\frac{|1-b| a}{\Delta} \int_{-\infty}^{+\infty} \frac{d u}{\rho(u)}+1\right] \int_{-\infty}^{+\infty} \varphi_{1}(s) d s \sum_{j=1}^{n} a_{j}[r+|| \bar{\Phi}|| \mid]^{\tau_{j}+\delta_{j}}[r+|| \bar{\Psi}||]^{\sigma_{j}+\gamma_{j}} \\
& \leq \max \left\{1, \frac{|1-a|+a}{|1-a|} \int_{-\infty}^{+\infty} \frac{d u}{\rho(u)}\right\}\left[\frac{|1-a| b}{\Delta} \int_{-\infty}^{+\infty} \frac{d u}{\rho(u)}+\frac{|1-a|}{\Delta} \int_{-\infty}^{+\infty} \frac{d u}{\rho(u)}\right. \\
& \left.+\frac{|1-b| a}{\Delta} \int_{-\infty}^{+\infty} \frac{d u}{\rho(u)}+1\right] \int_{-\infty}^{+\infty} \varphi_{1}(s) d s \sum_{j=1}^{n} a_{j}[r+A]^{\tau_{j}+\delta_{j}+\sigma_{j}+\gamma_{j}} \\
& \leq \max \left\{1, \frac{|1-a|+a}{|1-a|} \int_{-\infty}^{+\infty} \frac{d u}{\rho(u)}\right\}\left[\frac{|1-a| b}{\Delta} \int_{-\infty}^{+\infty} \frac{d u}{\rho(u)}+\frac{|1-a|}{\Delta} \int_{-\infty}^{+\infty} \frac{d u}{\rho(u)}\right. \\
& \left.+\frac{|1-b| a}{\Delta} \int_{-\infty}^{+\infty} \frac{d u}{\rho(u)}+1\right] \int_{-\infty}^{+\infty} \varphi_{1}(s) d s(r+A)^{\sigma} \sum_{j=1}^{n} a_{j} A^{\tau_{j}+\delta_{j}+\sigma_{j}+\gamma_{j}-\sigma} \\
& \leq M(r+A)^{\sigma} .
\end{aligned}
$$

Similarly, we have

$$
\| T_{2}(x, y)-\bar{\Psi}|| \leq \max \left\{1, \frac{|1-c|+c}{|1-c|} \int_{-\infty}^{+\infty} \frac{d u}{\varrho(u)}\right\} \sup _{t \in \mathbb{R}} \varrho(t)\left|\left(T_{2}(x, y)\right)^{\prime}(t)-\bar{\Psi}^{\prime}(t)\right| \leq M(r+A)^{\sigma} .
$$

It follows that $\|(T(x, y))-(\bar{\Phi}, \bar{\Psi})\| \leq M(r+A)^{\sigma}$.

Case 1. $\sigma \in[0,1)$. It is easy to see that there exists $r_{0}>0$ such that $M\left(r_{0}+A\right)^{\sigma} \leq r_{0}$. Choose $\Omega_{0}=\left\{(x, y) \in E:\|(x, y)-(\bar{\Phi}, \bar{\Psi})\| \leq r_{0}\right\}$. We have $T\left(\Omega_{0}\right) \subset \Omega_{0}$. Then $T$ has a fixed point $(x, y) \in \Omega_{0}$. So $(x, y) \in P$ is a positive solution of $\operatorname{BVP}(1.5)$.

Case 2. $\sigma=1$. (3.13) implies $M<1$. Choose $r_{0} \geq \frac{M A}{1-M}$ and $\Omega_{0}=\{(x, y) \in E: \|(x, y)-$ $\left.(\bar{\Phi}, \bar{\Psi}) \| \leq r_{0}\right\}$. We have $T\left(\Omega_{0}\right) \subset \Omega_{0}$. Then $T$ has a fixed point $(x, y) \in \Omega_{0}$. So $(x, y) \in P$ is a positive solution of $\operatorname{BVP}(1.5)$.

Case 3. $\sigma>1$. Choose $r_{0}=\frac{A}{\sigma-1}$ and $\Omega_{0}=\left\{(x, y) \in E:\|(x, y)-(\bar{\Phi}, \bar{\Psi})\| \leq r_{0}\right\}$. From (3.13), we have

$$
M\left(r_{0}+A\right)^{\sigma}=M\left(\frac{A}{\sigma-1}+A\right)^{\sigma} \leq r_{0} .
$$

Then $T\left(\Omega_{0}\right) \subset \Omega_{0}$. Then $T$ has a fixed point $(x, y) \in \Omega_{0}$. So $(x, y) \in P$ is a positive solution of $\operatorname{BVP}(1.5)$.

The proof of Theorem 3.2 is complete.

\section{An example}

In this section, we present two examples to illustrate the main theorems. 
Example 4.1. Consider the following boundary value problem of second order differential equation on the whole line

$$
\left\{\begin{array}{l}
{\left[\rho(t) x^{\prime}(t)\right]^{\prime}+f\left(t, x(t), y(t), x^{\prime}(t), y^{\prime}(t)\right)=0, a . e ., t \in \mathbb{R}} \\
{\left[\varrho(t) y^{\prime}(t)\right]^{\prime}+g\left(t, x(t), y(t), x^{\prime}(t), y^{\prime}(t)\right)=0, a . e ., t \in \mathbb{R}} \\
\lim _{t \rightarrow-\infty} x(t)-\frac{7}{5} x(-1)=0 \\
\lim _{t \rightarrow+\infty} x(t)-\frac{1}{6} x(1)=0 \\
\lim _{t \rightarrow-\infty} y(t)-\frac{1}{5} y(-1)=0 \\
\lim _{t \rightarrow+\infty} \varrho(t) y^{\prime}(t)-\frac{1}{25} \varrho(1) y^{\prime}(1)=0
\end{array}\right.
$$

where

$$
\begin{aligned}
& \rho(t)=\left\{\begin{array}{l}
\frac{1}{t^{2}}, t \in[-1,0) \bigcup(0,1], \\
t^{2},|t| \geq 1,
\end{array} \varrho(t)=\left\{\begin{array}{l}
\frac{1}{t^{4}}, t \in[-1,0) \bigcup(0,1], \\
t^{4},|t| \geq 1,
\end{array}\right.\right. \\
& f(t, x, y, u, v)=\varphi(t)+e^{-t^{2}}\left(a_{1}|x(t)|^{\alpha}+a_{2}|y(t)|^{\beta}+a_{3}\left[\rho(t) x^{\prime}(t)\right]^{\gamma}+a_{4}\left[\varrho(t) y^{\prime}(t)\right]^{\delta}\right), \\
& g(t, x, y, u, v)=\psi(t)+e^{-4 t^{2}}\left(b_{1}|x(t)|^{\alpha}+b_{2}|y(t)|^{\beta}+b_{3}\left[\rho(t) x^{\prime}(t)\right]^{\gamma}+b_{4}\left[\varrho(t) y^{\prime}(t)\right]^{\delta}\right), \\
& \varphi_{1}(t)=e^{-t^{2}}, \quad \psi_{1}(t)=e^{-4 t^{2}}, \quad \varphi(t)=e^{-4 t^{2}}, \quad \psi(t)=e^{-t^{2}} .
\end{aligned}
$$

Then $\operatorname{BVP}(4.14)$ has at least one positive solution if $\sigma=\max \{\alpha, \beta, \gamma, \delta\}$ satisfies

$$
\sigma \in[0,1) \text { or } \sigma=1 \text { with } M_{0}<1 \text { or } \sigma>1 \text { with } \frac{\sigma^{\sigma} A^{\sigma-1}}{(\sigma-1)^{\sigma-1}} \leq \frac{1}{M_{0}}
$$

where $M_{0}$ is defined by

$$
\begin{aligned}
M_{0}= & \frac{1695 \sqrt{\pi}}{2}\left[565^{\alpha-\sigma}\left(a_{1}+b_{1}\right)\left(\frac{3 \sqrt{\pi}}{2}\right)^{\alpha-\sigma}+565^{\beta-\sigma}\left(a_{2}+b_{2}\right)\left(\frac{3 \sqrt{\pi}}{2}\right)^{\beta-\sigma}\right. \\
& \left.+565^{\gamma-\sigma}\left(a_{3}+b_{3}\right)\left(\frac{3 \sqrt{\pi}}{2}\right)^{\gamma-\sigma}+565^{\delta-\sigma}\left(a_{4}+b_{4}\right)\left(\frac{3 \sqrt{\pi}}{2}\right)^{\delta-\sigma}\right] .
\end{aligned}
$$

Proof. Corresponding to $\operatorname{BVP}(1.5)$, we have $\xi=-1, \eta=1$ and $a=\frac{7}{5}, b=\frac{1}{6}, c=\frac{1}{5}, d=\frac{1}{25}$. Then

$$
\begin{aligned}
& \int_{-\infty}^{+\infty} \frac{d u}{\rho(u)}=\frac{8}{3}, \int_{-\infty}^{+\infty} \frac{d u}{\varrho(u)}=\frac{16}{15}, \\
& \|\varphi\|_{1}=\left\|\psi_{1}\right\|_{1}=\frac{\sqrt{\pi}}{2},\|\psi\|_{1}=\left\|\varphi_{1}\right\|_{1}=\sqrt{\pi}, \\
& (1-a) \int_{-\infty}^{+\infty} \frac{d u}{\rho(u)}+a \int_{-\infty}^{\xi} \frac{d u}{\rho(u)}=-\frac{2}{5} \times \frac{8}{3}+\frac{7}{5} \times 1=\frac{1}{3}, \\
& \int_{\eta}^{+\infty} \frac{d u}{\rho(u)}+(1-b) \int_{-\infty}^{\eta} \frac{d u}{\rho(u)}=\frac{11}{6}, \\
& \Delta=:(1-a) \int_{-\infty}^{+\infty} \frac{d u}{\rho(u)}-(1-a) b \int_{-\infty}^{\eta} \frac{d u}{\rho(u)}+a(1-b) \int_{-\infty}^{\xi} \frac{d u}{\rho(u)}=\frac{1}{30}, \\
& c=\frac{1}{5}, \quad d=\frac{1}{25} .
\end{aligned}
$$


So (2.7), (2.8.1), (2.8.2), (2.8.3) and (2.8) hold. By the definition of $\bar{\Phi}$ and $\bar{\Psi}$, we get

$$
\begin{aligned}
\|\bar{\Phi}\| \leq & \max \left\{1, \frac{|1-a|+a}{|1-a|} \int_{-\infty}^{+\infty} \frac{d u}{\rho(u)}\right\}\left[\frac{|1-a| b}{\Delta} \int_{-\infty}^{+\infty} \frac{d u}{\rho(u)}+\frac{|1-a|}{\Delta} \int_{-\infty}^{+\infty} \frac{d u}{\rho(u)}\right. \\
& \left.+\frac{|1-b| a}{\Delta} \int_{-\infty}^{+\infty} \frac{d u}{\rho(u)}+1\right] \int_{-\infty}^{+\infty} \varphi(s) d s \leq 565 \int_{-\infty}^{+\infty} \varphi(s) d s \leq 565\left(\|\varphi\|_{1}+\|\psi\|_{1}\right), \\
\|\bar{\Psi}\| \leq & \max \left\{1, \frac{1}{1-c} \int_{-\infty}^{+\infty} \frac{d u}{\varrho(u)}\right\}\left(\frac{1+d}{1-d}+1\right)\|\psi\|_{1} \leq 565\left(\|\varphi\|_{1}+\|\psi\|_{1}\right) .
\end{aligned}
$$

By direct computation, we have

$$
\begin{aligned}
& A=\frac{1695 \sqrt{\pi}}{2}=565\left(\|\varphi\|_{1}+\|\psi\|_{1}\right) \geq \max \{\|\bar{\Phi}\|,\|\bar{\Psi}\|\}, \sigma=\max \{\alpha, \beta, \gamma, \delta\}, \\
& M=\max \left\{5 6 5 \| \varphi _ { 1 } \| _ { 1 } \left[565^{\alpha-\sigma} a_{1}\left(\|\varphi\|_{1}+\|\psi\|_{1}\right)^{\alpha-\sigma}+565^{\beta-\sigma} a_{2}\left(\|\varphi\|_{1}+\|\psi\|_{1}\right)^{\beta-\sigma}\right.\right. \\
& \left.\quad+565^{\gamma-\sigma} a_{3}\left(\|\varphi\|_{1}+\|\psi\|_{1}\right)^{\gamma-\sigma}+565^{\delta-\sigma} a_{4}\left(\|\varphi\|_{1}+\|\psi\|_{1}\right)^{\delta-\sigma}\right], \\
& \quad \frac{238}{3}\left\|\psi_{1}\right\|_{1}\left[\left(\frac{238}{3}\right)^{\alpha-\sigma} b_{1}\left(\|\varphi\|_{1}+\|\psi\|_{1}\right)^{\alpha-\sigma}+\left(\frac{238}{3}\right)^{\beta-\sigma} b_{2}\left(\|\varphi\|_{1}+\|\psi\|_{1}\right)^{\beta-\sigma}\right. \\
& \left.\left.+\left(\frac{238}{3}\right)^{\gamma-\sigma} b_{3}\left(\|\varphi\|_{1}+\|\psi\|_{1}\right)^{\gamma-\sigma}+\left(\frac{238}{3}\right)^{\delta-\sigma} b_{4}\left(\|\varphi\|_{1}+\|\psi\|_{1}\right)^{\delta-\sigma}\right]\right\} \\
& \leq 565\left(\left\|\varphi_{1}\right\|\left\|_{1}+\right\| \psi_{1} \|_{1}\right)\left[565^{\alpha-\sigma}\left(a_{1}+b_{1}\right)\left(\|\varphi\|_{1}+\|\psi\|_{1}\right)^{\alpha-\sigma}+565^{\beta-\sigma}\left(a_{2}+b_{2}\right)\left(\|\varphi\|_{1}+\|\psi\|_{1}\right)^{\beta-\sigma}\right. \\
& \left.+565^{\gamma-\sigma}\left(a_{3}+b_{3}\right)\left(\|\varphi\|_{1}+\|\psi\|_{1}\right)^{\gamma-\sigma}+565^{\delta-\sigma}\left(a_{4}+b_{4}\right)\left(\|\varphi\|_{1}+\|\psi\|_{1}\right)^{\delta-\sigma}\right] \\
& \leq \frac{1695 \sqrt{\pi}}{2}\left[565^{\alpha-\sigma}\left(a_{1}+b_{1}\right)\left(\frac{3 \sqrt{\pi}}{2}\right)^{\alpha-\sigma}+565^{\beta-\sigma}\left(a_{2}+b_{2}\right)\left(\frac{3 \sqrt{\pi}}{2}\right)^{\beta-\sigma}\right. \\
& \left.+565^{\gamma-\sigma}\left(a_{3}+b_{3}\right)\left(\frac{3 \sqrt{\pi}}{2}\right)^{\gamma-\sigma}+565^{\delta-\sigma}\left(a_{4}+b_{4}\right)\left(\frac{3 \sqrt{\pi}}{2}\right)^{\delta-\sigma}\right] .
\end{aligned}
$$

Then Theorem 3.2 implies that BVP(4.14) has at least one positive solution $(x, y)$ if $(3.13)$ holds. Since (4.15) implies that (3.13), then BVP(4.14) has at least one positive solution $(x, y)$ if $(4.15)$ holds.

Acknowledgement. The authors would like to thank the referees and the editors for their careful reading and some useful comments on improving the presentation of this paper.

\section{References}

[1] R.P. Agarwal, Boundary value problems for higher order differential equations, World Scientific, Singapore, 1986. 
[2] C. Avramescu, C. Vladimirescu, Existence of Homoclinic solutions to a nonlinear second order ODE, Dynamics of continuous, discrete and impulsive systems, Ser. A, Math Anal. 15 (2008), 481-491.

[3] C. Avramescu, C. Vladimirescu, Existence of solutions to second order ordinary differential equations having finite limits at $\pm \infty$, Electronic Journal of Differential Equations 18 (2004), $1-12$.

[4] C. Avramescu and C. Vladimirescu, Limits of solutions of a perturbed linear differential equation, E. J. Qualitative Theory of Diff. Equ. 3 (2002), 1-11.

[5] A. A. Boichuk, O. A. Pokutnyi, Dichotomy and boundary value problems on the whole line, Chaotic Modeling and Simulation (CMSIM) 2 (2013), 247-255.

[6] B. Bianconi, F. Papalini, Non-autonomous boundary value problems on the real line, Discrete and continuous dynamical systems 15 (2006), 759-776.

[7] A. Calamai, Heteroclinic solutions of boundary value problems on the real line involving singular Ф-Laplacian operators, Journal of Mathematical Analysis and Applications 378 (2011), 667679 .

[8] A. Cabada, J.A. Cid, Heteroclinic solutions for non-autonomous boundary value problems with singular $\Phi$-Laplacian operators, Discrete Contin. Dyn. Syst. 2009, Dynamical Systems, Differential Equations and Applications. 7th AIMS Conference, suppl., 118-122.

[9] G. Cupini, C. Marcelli, F. Papalini, Heteroclinic solutions of boundary value problems on the real line involving general nonlinear differential operators, Differ. Integral Equ. 24(7-8) (2011), 619-644.

[10] G. Cupini, C. Marcelli, F. Papalini, On the solvability of a boundary value problem on the real line, Bound. Value Probl. 2011, 26 (2011).

[11] Q. Dai, Entire positive solutions for in-homogeneous semilinear elliptic systems, Glasgow Math. Journal 47 (2005), 97-114.

[12] Q. Dai, Y. Fu, Nondegeneracy and uniqueness of positive solutions for Robin problem of second order ordinary differential equations and its applications, Journal of Diff. Equs. 241 (2007), 3671.

[13] Q. Dai, C. C. Tisdell, Nondegeneracy of positive solutions to homogeneous second-order differential systems and its applications, Acta Mathematica Scientia 29(B)(2) (2009), 435-446.

[14] K. Deimling, Nonlinear Functional Analysis, Springer, Berlin, Germany, 1985.

[15] F. Y. Deren, N. A. Hamal, Second-order boundary-value problems with integral boundary conditions on the real line, Electronic Journal of Differential Equations 19 (2014), 1-13.

[16] W. Ge, Boundary Value Problems for Ordinary Differential Equations (in Chinese), Science Press, Beijing, 2007. 
[17] P. Han, Z. Liu, Multiple positive solutions of strongly indefinite systems with critical Sobolev exponents and data that changes sign, Nonlinear Anal. 58(1/2) (2004), 229-243.

[18] V. A. Il'in, E. I. Moiseev, Nonlocal boundary-value problem of the second kind for a SturmLiouville operator, Differential Equations 23 (1987), 979-987.

[19] B. Liu, Positive solutions of a nonlinear four-point boundary value problems in Banach spaces, J. Math. Anal. Appl. 305 (2005), 253-276.

[20] Y. Liu, Multiple positive solutions to mixed boundary value problems for singular ordinary differential equations on the whole line, Nonlinear Analysis: Modelling and Control 17 (4) (2012), 460-480.

[21] Y. Liu, Existence of multiple positive solutions of p-Laplacian boundary value problems, Math. Slovak. 57 (2007), 225-242.

[22] Y. Liu, Solutions to second order non-homogeneous multi-point BVPs using a fixed-point theorem, Electronic Journal of Differential Equations 96 (2008), 1-52.

[23] C. Marcelli, Existence of solutions to some boundary-value problems governed by general nonlinear non-autonomous differential operators, Electron. J. Differ. Equ. 2012, 171 (2012).

[24] C. Marcelli, The role of boundary data on the solvability of some equations involving nonautonomous nonlinear differential operators, Boundary Value Problems 2013, 2013: 252.

[25] C. Marcelli, F. Papalini, Heteroclinic connections for fully non-linear non-autonomous secondorder differential equations, Journal of Differential Equations 241 (2007), 160-183.

[26] K. R. Prasad, A. K. Rao, S. N. Rao, Existence of positive solutions for the system of higher order two-point boundary value problems, Proceedings of the Indian Academy of Sciences Mathematical Sciences 122 (2012), 139-152.

[27] C. G. Philos, I. K. Purnaras, A boundary value problem on the whole line to second order nonlinear differential equations, Georgian Mathematical Journal 17 (2010), 241-252.

[28] H. Zou, A priori estimates for semilinear elliptic system without variational structure and their applications, Math. Ann. 323 (2002), 713-735. 